\title{
Measuring the Ultimate Halo Mass of Galaxy Clusters: Redshifts and Mass Profiles from the Hectospec Cluster Survey (HeCS)
}

Kenneth J. Rines

Follow this and additional works at: https://cedar.wwu.edu/physicsastronomy_facpubs

Part of the Astrophysics and Astronomy Commons

\section{Recommended Citation}

Rines, Kenneth J., "Measuring the Ultimate Halo Mass of Galaxy Clusters: Redshifts and Mass Profiles from the Hectospec Cluster Survey (HeCS)" (2013). Physics \& Astronomy. 34.

https://cedar.wwu.edu/physicsastronomy_facpubs/34 


\title{
MEASURING THE ULTIMATE HALO MASS OF GALAXY CLUSTERS: REDSHIFTS AND MASS PROFILES FROM THE HECTOSPEC CLUSTER SURVEY (HeCS)
}

\author{
Kenneth Rines $^{1,2}$, Margaret J. Geller ${ }^{2}$, Antonaldo Diaferio ${ }^{3,4}$, and Michael J. Kurtz ${ }^{2}$ \\ ${ }^{1}$ Department of Physics \& Astronomy, Western Washington University, Bellingham, WA 98225, USA; kenneth.rines@wwu.edu \\ ${ }^{2}$ Smithsonian Astrophysical Observatory, 60 Garden Street, MS 20, Cambridge, MA 02138, USA \\ ${ }^{3}$ Dipartimento di Fisica, Università di Torino, Torino, Italy; diaferio@ph.unito.it \\ ${ }^{4}$ Istituto Nazionale di Fisica Nucleare (INFN), Sezione di Torino, Torino, Italy \\ Received 2012 September 17; accepted 2013 January 29; published 2013 March 20
}

\begin{abstract}
The infall regions of galaxy clusters represent the largest gravitationally bound structures in a $\Lambda$ CDM universe. Measuring cluster mass profiles into the infall regions provides an estimate of the ultimate mass of these halos. We use the caustic technique to measure cluster mass profiles from galaxy redshifts obtained with the Hectospec Cluster Survey (HeCS), an extensive spectroscopic survey of galaxy clusters with MMT/Hectospec. We survey 58 clusters selected by X-ray flux at $0.1<z<0.3$. The survey includes 22,680 unique MMT/Hectospec redshifts for individual galaxies; 10,145 of these galaxies are cluster members. For each cluster, we acquired high signalto-noise spectra for $\sim 200$ cluster members and a comparable number of foreground/background galaxies. The cluster members trace out infall patterns around the clusters. The members define a very narrow red sequence. We demonstrate that the determination of velocity dispersion is insensitive to the inclusion of bluer members (a small fraction of the cluster population). We apply the caustic technique to define membership and estimate the mass profiles to large radii. The ultimate halo mass of clusters (the mass that remains bound in the far future of a $\Lambda \mathrm{CDM}$ universe) is on average $(1.99 \pm 0.11) M_{200}$, a new observational cosmological test in essential agreement with simulations. Summed profiles binned in $M_{200}$ and in $L_{X}$ demonstrate that the predicted Navarro-Frenk-White form of the density profile is a remarkably good representation of the data in agreement with weak lensing results extending to large radius. The concentration of these summed profiles is also consistent with theoretical predictions.
\end{abstract}

Key words: cosmology: observations - galaxies: clusters: general - galaxies: kinematics and dynamics

Online-only material: color figures, extended figures, machine-readable tables

\section{INTRODUCTION}

Clusters of galaxies are the most massive virialized systems in the universe. Clusters are surrounded by infall regions where galaxies are bound to the cluster but are not in dynamical equilibrium in the cluster potential. If dark energy behaves like a cosmological constant, cluster infall regions are the largest gravitationally bound structures in the universe. Thus, measuring cluster mass profiles at large radii provides an estimate of the ultimate halo mass of these systems (Nagamine \& Loeb 2003; Busha et al. 2005; Dünner et al. 2006).

The Cluster and Infall Region Nearby Survey (CAIRNS) pioneered the detailed observational study of cluster infall regions. CAIRNS studied nine nearby galaxy clusters and their infall regions with extensive spectroscopy (Rines et al. 2003, 2005) and near-infrared photometry from the Two-Micron All-Sky Survey (Rines et al. 2004). The nine CAIRNS clusters display a characteristic trumpet-shaped pattern in radius-redshift phase space diagrams. These patterns were first predicted for simple spherical infall onto clusters (Kaiser 1987; Regös \& Geller 1989), but later work showed that these patterns reflect the dynamics of the infall region (Diaferio \& Geller 1997; Diaferio 1999, hereafter DG and D99). The Cluster Infall Regions in the Sloan Digital Sky Survey (Rines \& Diaferio 2006, hereafter CIRS) project extended this analysis to $72 \mathrm{X}$-ray-selected clusters in the Sloan Digital Sky Survey (SDSS; Stoughton et al. 2002). CIRS showed that these infall patterns are ubiquitous in nearby $\mathrm{X}$-ray clusters.

Using numerical simulations, DG and D99 showed that the amplitude of the caustics is a measure of the escape velocity from the cluster; identification of the caustics therefore allows a determination of the mass profile of the cluster on scales $\lesssim 10 h^{-1}$ Mpc. In particular, nonparametric measurements of caustics yield cluster mass profiles accurate to $\sim 50 \%$ on scales $\lesssim 10 h^{-1}$ Mpc when applied to Coma-size clusters extracted from cosmological simulations. Serra et al. (2011) confirm these results for clusters across a broader mass range and they show that the dominant source of uncertainty in individual cluster mass profiles is projection effects from departures from spherical symmetry. The caustic technique assumes only that galaxies trace the velocity field. Indeed, simulations suggest that little or no velocity bias exists on linear and mildly nonlinear scales (Kauffmann et al. 1999a, 1999b; Diemand et al. 2004; Faltenbacher et al. 2005). This conclusion is supported observationally by the excellent agreement between the cluster virial mass function and other cosmological probes (Rines et al. 2008).

CAIRNS and CIRS showed that caustic masses of clusters agree well with mass estimates from both X-ray observations and Jeans' analysis at small radii (Rines et al. 2003, CIRS). Łokas \& Mamon (2003) confirm that the mass of Coma estimated from higher moments of the velocity distribution agrees well with the caustic mass estimate (Geller et al. 1999).

The caustic technique provides an estimate of the mass profile of clusters. For instance, CAIRNS and CIRS showed that cluster mass profiles fall off more steeply at large radii than an isothermal sphere. Thus, caustic mass profiles probe the structure of dark matter halos, and these profiles can be compared to those determined from gravitational lensing (e.g., Umetsu et al. 2011; Geller et al. 2013). 
At large radii, neither galaxies nor intracluster gas should be in equilibrium, thus invalidating the use of the virial theorem or hydrostatic equilibrium at these radii. The caustic technique and gravitational lensing are the only cluster mass estimators that do not rely on the equilibrium assumption. Gravitational lensing is necessarily contaminated by line-of-sight structure unrelated to the cluster; this contamination becomes larger at larger radii (e.g., Hoekstra et al. 2011). Despite this potential difficulty, Diaferio et al. (2005) showed that caustic masses agree with weak lensing masses in three clusters at moderate redshift.

CAIRNS and CIRS showed that infall patterns are well defined in observations of nearby massive clusters. In fact, the infall patterns or "caustics" have significantly higher contrast in the CAIRNS observations than in the simulations of DG and D99. The CAIRNS and CIRS clusters are fairly massive clusters and generally have little surrounding large-scale structure (but see Rines et al. 2001, 2002).

One might suspect that the presence of infall patterns is limited to massive, isolated clusters. However, other investigators have found infall patterns around the Fornax Cluster (Drinkwater et al. 2001), the Shapley Supercluster (Reisenegger et al. 2000), an ensemble cluster comprised of poor clusters in the Two Degree Field Galaxy Redshift Survey (Biviano \& Girardi 2003), and even the galaxy group associated with NGC 5846 (Mahdavi et al. 2005). Caustics are also identifiable in X-ray groups in SDSS (Rines \& Diaferio 2010).

The presence of caustics in all nine CAIRNS clusters and in all 72 CIRS clusters suggests that they are ubiquitous in nearby, massive clusters. Because clusters and especially cluster infall regions form quite late in the evolution of structure, infall patterns evolve even at modest redshifts (see an animation in Geller et al. 2011). Diaferio et al. (2005) showed that infall patterns exist in three clusters at moderate redshift, although these clusters were not carefully selected. Similarly, Lemze et al. (2009) showed that the caustic mass profile of A1689 $(z=0.18)$ agrees with the mass profile determined from a joint analysis of $\mathrm{X}$-ray and lensing data.

Inspired by these results, we conducted a systematic survey of infall regions at $z \approx 0.2$. The Hectospec Cluster Survey (HeCS), includes MMT/Hectospec spectra of large samples of galaxies in the infall regions of X-ray-selected clusters at $z=0.1-0.3$. $\mathrm{HeCS}$ is the first systematic spectroscopic survey of cluster infall regions at $z \gtrsim 0.1$.

Spectroscopic data for clusters at moderate redshift can test the robustness of scaling relations between different cluster observables that correlate with cluster mass. For instance, the integrated Sunyaev-Zeldovich decrement $Y_{\mathrm{SZ}}$ has small scatter in simulations (Hallman et al. 2006), as does the X-ray observable $Y_{X}=M_{\text {gas }} T_{X}$ (Kravtsov et al. 2006). The $Y_{X}$ parameter also seems to have low scatter in observations, although the estimated masses and $Y_{X}$ are derived from the same data (Nagai et al. 2007). Rines et al. (2010) studied the relation between $Y_{\mathrm{SZ}}$ and the dynamics of galaxies in a sample of 15 clusters. The dynamical properties correlate strongly with increasing $Y_{\mathrm{SZ}}$ but with significant scatter.

We describe the data and the cluster sample in Section 2. In Section 3, we review the caustic technique, use it to estimate the cluster mass profiles, and estimate the ultimate halo masses of clusters. To mitigate systematic uncertainties due to projection effects, we analyze ensemble clusters in Section 4 and compare the ensemble mass profiles with the theoretical Navarro-Frenk-White (NFW) profile (Navarro et al. 1997) and with profiles determined from gravitational lensing. We discuss the color distribution of cluster members in Section 5 and show that our red-sequence selection does not bias our dynamical measurements. We discuss our results and conclude in Section 6. We assume $H_{0}=100 h \mathrm{~km} \mathrm{~s}^{-1}, \Omega_{m}=0.3, \Omega_{\Lambda}=$ 0.7 throughout. We measure cluster masses $M_{\Delta}$, defined as the mass enclosed within the radius $r_{\Delta}$ that encloses an average density of $\Delta \rho_{c}(z)$, where $\rho_{c}(z)=3 H_{0}^{2} E^{2}(z) / 8 \pi G$ is the critical density at redshift $z$ and $E^{2}(z)=\Omega_{m}(1+z)^{3}+\Omega_{\Lambda}+\left(1-\Omega_{m}-\right.$ $\left.\Omega_{\Lambda}\right)(1+z)^{2}$.

\section{THE HeCS CLUSTER SAMPLE}

We construct the HeCS sample to take advantage of two large-area public surveys: the SDSS (Stoughton et al. 2002) and the ROSAT All-Sky Survey (RASS; Voges et al. 1999). In particular, we utilize existing X-ray cluster catalogs based on RASS data to define a flux-limited cluster sample. We then match these systems to the imaging footprint of the SDSS Data Release 6 (DR6; Adelman-McCarthy et al. 2008). The accurate SDSS multicolor photometry enables selection of candidate cluster members using the red-sequence technique (e.g., Gladders \& Yee 2000).

We obtained MMT/Hectospec spectroscopy of 400-550 candidate members per cluster (for three clusters, we obtained more than 550 spectra). Hectospec redshifts enable robust membership classification and estimates of the virial masses of the clusters. The wide field-of-view of Hectospec allows us to simultaneously probe the virial and infall regions of these clusters.

The earlier CIRS project used spectroscopic data from the SDSS (Stoughton et al. 2002) to study clusters at $z \leqslant 0.1$ identified in X-ray cluster catalogs based on the RASS. At $z>$ 0.1 , the SDSS redshift survey is not dense enough for accurate assessment of cluster masses.

\subsection{Sloan Digital Sky Survey}

The SDSS (Stoughton et al. 2002) is a wide-area photometric and spectroscopic survey at high Galactic latitudes. The Sixth Data Release (DR6) of SDSS includes $8417 \mathrm{deg}^{2}$ of imaging data (Adelman-McCarthy et al. 2008).

From a comparison of SDSS with the Millennium Galaxy Catalogue, Cross et al. (2004) conclude that there is a photometric incompleteness of $\sim 7 \%$ due to galaxies misclassified as stars or otherwise missed by the SDSS photometric pipeline. For our purposes, the incompleteness is not important provided sufficient numbers of cluster galaxies do have spectra. Further, the cluster galaxies we focus on here are intrinsically luminous and have higher surface brightnesses than less luminous galaxies. Thus, the photometric incompleteness is probably somewhat smaller for these galaxies.

The spectroscopic limit of the main galaxy sample of SDSS is $r=17.77$ after correcting for Galactic extinction (Strauss et al. 2002). CAIRNS and CIRS show that infall patterns are easily detectable in clusters sampled to about $M^{*}+1$. For SDSS, this limit is reached at $z \sim 0.1$; deeper spectroscopic surveys are required to study more distant clusters. Studying cluster infall regions at $z \sim 0.2$ requires spectroscopy to a limit of $r \sim 21$.

\subsection{X-Ray Cluster Surveys}

We select the HeCS clusters from X-ray catalogs based on the RASS (Voges et al. 1999). RASS is a shallow survey, but it is sufficiently deep to include nearby, massive clusters. 


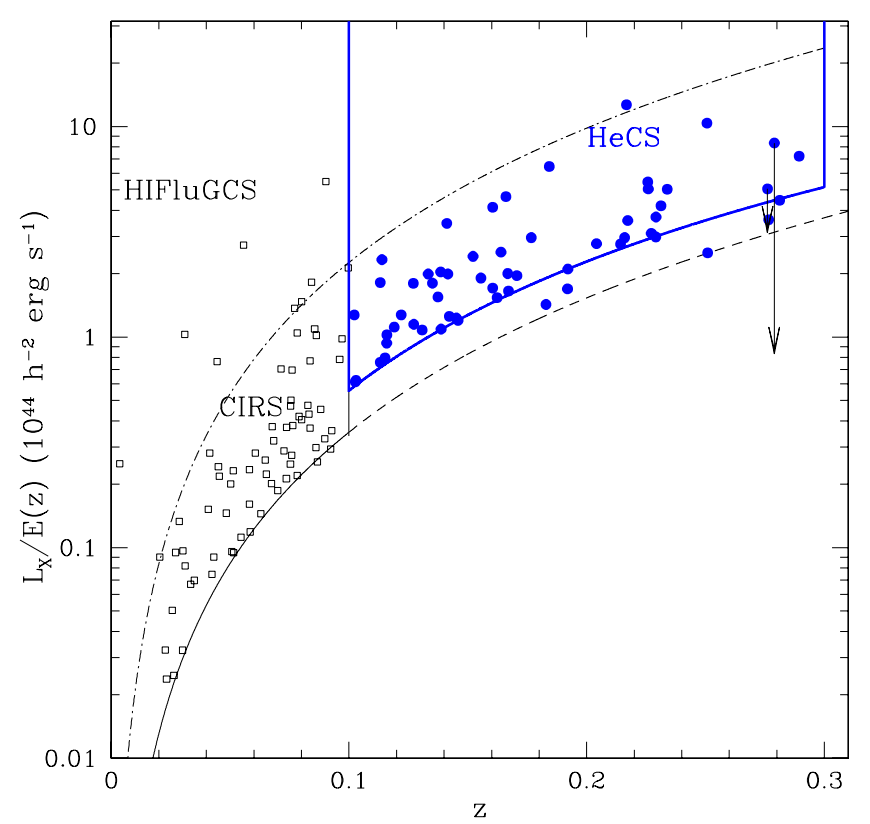

Figure 1. Redshift vs. X-ray luminosity $(0.1-2.4 \mathrm{keV})$ for X-ray clusters from CIRS (small open squares) and HeCS clusters (filled blue circles) contained in the SDSS DR6 imaging survey region. The blue solid lines show the selection of the HeCS sample: redshifts $0.1<z<0.3$ and a flux limit of $f_{X}>5 \times 10^{-12} \mathrm{erg} \mathrm{s}^{-1} \mathrm{~cm}^{-2}$. Four HeCS clusters are from a subsample of clusters with R.A. $>17^{\mathrm{h}}$ and flux $f_{X}>3 \times 10^{-12} \mathrm{erg} \mathrm{s}^{-1} \mathrm{~cm}^{-2}$ (the same flux limit as CIRS). The lower solid line shows the flux and redshift limits of the CIRS cluster sample. The dash-dotted line shows the flux limit $\left(2 \times 10^{-11} \mathrm{erg} \mathrm{s}^{-1} \mathrm{~cm}^{-2}\right)$ of the HiFluGCS sample.

(A color version of this figure is available in the online journal.)

RASS covers virtually the entire sky and is thus the most complete X-ray cluster survey for nearby clusters. The flux limits of RASS-based surveys are $\approx 3 \times 10^{-12} \mathrm{erg} \mathrm{s}^{-1} \mathrm{~cm}^{-2}$ in the ROSAT band (Ebeling et al. 1998; Böhringer et al. 2000, 2004).

We restrict our study to systems with $0.10 \leqslant z \leqslant 0.30$. The flux-limited HeCS sample consists of the 53 clusters from the Bright Cluster Survey (BCS; Ebeling et al. 1998) and ROSAT-ESO FLux-LimitEd X-ray cluster survey (REFLEX; Böhringer et al. 2004) catalogs within the SDSS DR6 photometric footprint and with $f_{X} \geqslant 5 \times 10^{-12} \mathrm{erg} \mathrm{s}^{-1} \mathrm{~cm}^{-2}$. Due to scheduling constraints on observing time, HeCS includes four additional clusters (A2187, A2396, A2631, and A2645) with $f_{X} \geqslant 3 \times 10^{-12} \mathrm{erg} \mathrm{s}^{-1} \mathrm{~cm}^{-2}$ : A2645 is in REFLEX and the others are from the extended BCS (eBCS; Ebeling et al. 2000). BCS splits A1758 into two components, neither of which alone would lie above our flux limit; however, the NORAS catalog (Böhringer et al. 2000) merges these components, yielding a flux above our flux limit. We also include an X-ray cluster, A750, that lies in the foreground of MS0906+1110 (see Section 3.6 for details). HeCS thus contains 58 clusters: 53 clusters in the flux-limited sample, four clusters with slightly smaller X-ray fluxes, and A750 (we do not count the subclusters of A1758 as individual clusters).

A689 has a large contribution from a central BL Lac; Giles et al. (2012) estimate that only $10 \%$ of the BCS luminosity is from the intracluster medium. The long arrow in Figure 1 shows that this cluster would not lie in the flux-limited sample with the corrected luminosity. Similarly, the luminosity of A $1758 \mathrm{~N}$ (the brighter of $\mathrm{A} 1758 \mathrm{~N} / \mathrm{S}$ ) alone would remove it from the flux-limited sample (short arrow in Figure 1).

Table 1 describes the basic properties of the HeCS clusters. $\mathrm{X}$-ray luminosities are in the rest-frame ROSAT band and are from the BCS and REFLEX catalogs. Figure 1 shows the (rest-frame) X-ray luminosities of the HeCS clusters compared to the HiFluGCS survey (Reiprich \& Böhringer 2002) and to our previous survey CIRS. HeCS contains systems with significantly larger $L_{X}$ than CIRS, although there is a substantial overlap at intermediate $L_{X}$, especially at $z=0.10-0.15$. The larger redshift limit of HeCS relative to CIRS probes a much larger volume: the HeCS volume is $\sim 10^{8} h^{-3} \mathrm{Mpc}^{3}$, a factor of $>10$ larger than the volume probed by CIRS. The number density of clusters declines with $L_{X}$ (e.g., Böhringer et al. 2002); a larger survey volume increases the sample of intrinsically more luminous systems.

\subsection{MMT/Hectospec Spectroscopy}

The Hectospec instrument (Fabricant et al. 2005) mounted on the MMT $6.5 \mathrm{~m}$ telescope is ideal for studying cluster infall regions at moderate redshift. Hectospec is a multiobject fiberfed spectrograph with 300 fibers deployable over a circular field-of-view with a diameter of $1^{\circ}$. One Hectospec pointing extends to a radius of $2.3(5.6) h^{-1} \mathrm{Mpc}$ at $z=0.1(0.3)$, so a single Hectospec pointing covers the entire virial region and extends well into the infall region.

Our observing strategy for $\mathrm{HeCS}$ was to identify the red sequence of cluster galaxies for each system and target primarily galaxies within 0.3 magnitudes of the red sequence. As shown in Section 5.1, the color selection is significantly broader than the actual red sequence of confirmed cluster members. Thus, the target selection includes primarily, but not exclusively, red-sequence galaxies. We use SDSS DR6 spectroscopic data to identify galaxies with existing redshifts and remove them from the target catalog. The galaxy targets have $r$ magnitudes of $r=16-21$. The Hectospec fiber assignment software xfitfibs ${ }^{5}$ allows the user to rank targets with priorities. We ranked galaxies primarily by their proximity to the cluster center and secondarily by apparent magnitude. This procedure yields a largely complete magnitude-limited sample of brighter galaxies supplemented by a more sparsely sampled selection of galaxies up to 1 mag fainter than the bright limit. The Appendix describes the target selection procedure in more detail.

The galaxy targets are relatively bright for Hectospec spectroscopy: we can obtain high-quality spectra with $3 \times 20$ minute exposures even under suboptimal observing conditions (e.g., poor seeing, thin clouds). Because Hectospec is a queue-scheduled instrument, this flexibility allows the HeCS fields to be observed under many observing conditions and improve the overall efficiency of the Hectospec queue. Observations were conducted primarily between 2007 June and 2009 February with a total of 10 nights of queue time. We also observed one additional Hectospec field in A2261 in 2011 May and seven additional fields in RXJ2129 in 2011 September. We observed additional fields in these clusters because they are part of the CLASH sample (Postman et al. 2012; Coe et al. 2012). The supplementary Hectospec fields targeted galaxies of all colors, enabling us to test the potential impact of our red-sequence selection on the estimates of dynamical parameters (Section 5.3).

Because the number of galaxies per cluster varies significantly, we adjusted the limiting magnitudes primarily to obtain large, nearly complete, samples to the faintest magnitude possible. Thus, the limiting absolute magnitude varies significantly from cluster to cluster.

\footnotetext{
5 https://www.cfa.harvard.edu/ john/xfitfibs/
} 
Table 1

HeCS Basic Properties

\begin{tabular}{|c|c|c|c|c|c|c|c|}
\hline \multirow[t]{2}{*}{ Name } & \multicolumn{2}{|c|}{ X-Ray Coordinates } & \multirow[t]{2}{*}{$z_{\odot}$} & \multirow{2}{*}{$\begin{array}{l}L_{X} / 10^{43} \\
\left(\mathrm{erg} \mathrm{s}^{-1}\right)\end{array}$} & \multirow[t]{2}{*}{ Catalog } & \multirow{2}{*}{$\begin{array}{c}\sigma_{p} \\
\left(\mathrm{~km} \mathrm{~s}^{-1}\right)\end{array}$} & \multirow[t]{2}{*}{$N_{m}$} \\
\hline & R.A. (J2000) & Decl. (J2000) & & & & & \\
\hline A267 & 28.1762 & 01.0125 & 0.2291 & 4.16 & $\mathrm{BCS}$ & $972_{-53}^{+63}$ & 226 \\
\hline Zw1478 & 119.9190 & 53.9990 & 0.1027 & 0.64 & $\mathrm{BCS}$ & $479_{-46}^{+66}$ & 82 \\
\hline A646 & 125.5470 & 47.1000 & 0.1273 & 1.22 & BCS & $653_{-51}^{+66}$ & 264 \\
\hline A655 & 126.3610 & 47.1320 & 0.1271 & 1.91 & BCS & $777_{-47}^{+58}$ & 315 \\
\hline A667 & 127.0190 & 44.7640 & 0.1452 & 1.32 & BCS & $645_{-58}^{+80}$ & 148 \\
\hline A689 & 129.3560 & 14.9830 & 0.2789 & 9.62 & $\mathrm{BCS}$ & $589_{-62}^{+91}$ & 163 \\
\hline A697 & 130.7362 & 36.3625 & 0.2812 & 5.15 & $\mathrm{BCS}$ & $1002_{-75}^{+97}$ & 185 \\
\hline A750 & 137.2469 & 11.0444 & 0.1640 & $\ldots$ & BCS* & $681_{-45}^{+56}$ & 225 \\
\hline MS0906 & 137.2832 & 10.9925 & 0.1767 & 3.23 & $\mathrm{BCS}$ & $664_{-62}^{+87}$ & 101 \\
\hline A773 & 139.4624 & 51.7248 & 0.2173 & 3.98 & $\mathrm{BCS}$ & $1110_{-70}^{+86}$ & 173 \\
\hline A795 & 141.0240 & 14.1680 & 0.1374 & 1.65 & BCS & $778_{-50}^{+61}$ & 179 \\
\hline Zw2701 & 148.1980 & 51.8910 & 0.2160 & 3.30 & BCS & $652_{-55}^{+74}$ & 93 \\
\hline A963 & 154.2600 & 39.0484 & 0.2041 & 3.07 & BCS & $956_{-64}^{+80}$ & 211 \\
\hline A980 & 155.6275 & 50.1017 & 0.1555 & 2.06 & BCS & $1033_{-59}^{+72}$ & 222 \\
\hline Zw3146 & 155.9117 & 04.1865 & 0.2894 & 8.38 & BCS & $858_{-75}^{+103}$ & 106 \\
\hline A990 & 155.9120 & 49.1450 & 0.1416 & 2.13 & BCS & $655_{-60}^{+82}$ & 91 \\
\hline Zw3179 & 156.4840 & 12.6910 & 0.1422 & 1.34 & BCS & $541_{-73}^{+122}$ & 69 \\
\hline A1033 & 157.9320 & 35.0580 & 0.1220 & 1.35 & BCS & $677_{-44}^{+55}$ & 191 \\
\hline A1068 & 160.1870 & 39.9510 & 0.1386 & 2.18 & BCS & $1028_{-81}^{+106}$ & 129 \\
\hline A1132 & 164.6160 & 56.7820 & 0.1351 & 1.92 & BCS & $749_{-61}^{+80}$ & 160 \\
\hline A1201 & 168.2287 & 13.4448 & 0.1671 & 1.79 & BCS & $683_{-53}^{+68}$ & 165 \\
\hline A1204 & 168.3324 & 17.5937 & 0.1706 & 2.13 & BCS & $532_{-46}^{+62}$ & 92 \\
\hline A1235 & 170.8040 & 19.6160 & 0.1030 & 0.65 & BCS & $584_{-47}^{+62}$ & 131 \\
\hline A1246 & 170.9912 & 21.4903 & 0.1921 & 2.31 & BCS & $906_{-57}^{+70}$ & 226 \\
\hline A1302 & 173.3070 & 66.3990 & 0.1152 & 0.84 & BCS & $650_{-48}^{+62}$ & 162 \\
\hline A1361 & 175.9170 & 46.3740 & 0.1159 & 0.99 & BCS & $512_{-47}^{+64}$ & 195 \\
\hline A1366 & 176.2020 & 67.4130 & 0.1160 & 1.08 & BCS & $616_{-48}^{+62}$ & 200 \\
\hline A1413 & 178.8260 & 23.4080 & 0.1412 & 3.71 & BCS & $856_{-68}^{+90}$ & 116 \\
\hline A 1423 & 179.3420 & 33.6320 & 0.2142 & 3.07 & BCS & $759_{-51}^{+64}$ & 230 \\
\hline A1437 & 180.1040 & 03.3490 & 0.1333 & 2.12 & BCS & $1233_{-81}^{+102}$ & 194 \\
\hline A1553 & 187.6959 & 10.5606 & 0.1668 & 2.17 & BCS & $867_{-51}^{+62}$ & 171 \\
\hline A1682 & 196.7278 & 46.5560 & 0.2272 & 3.48 & BCS & $996_{-65}^{+80}$ & 151 \\
\hline A1689 & 197.8750 & -1.3353 & 0.1842 & 7.06 & REF & $1197_{-65}^{+78}$ & 210 \\
\hline A1758 & 203.1796 & 50.5496 & 0.2760 & 5.82 & $\mathrm{BCS}$ & $674_{-69}^{+99}$ & 143 \\
\hline A1763 & 203.8257 & 40.9970 & 0.2312 & 4.72 & BCS & $1261_{-68}^{+81}$ & 237 \\
\hline A1835 & 210.2595 & 02.8801 & 0.2506 & 11.77 & BCS & $1151_{-66}^{+80}$ & 219 \\
\hline A1902 & 215.4226 & 37.2958 & 0.1623 & 1.67 & BCS & $784_{-56}^{+71}$ & 130 \\
\hline A1918 & 216.3420 & 63.1830 & 0.1388 & 1.17 & BCS & $545_{-54}^{+76}$ & 80 \\
\hline A1914 & 216.5068 & 37.8271 & 0.1660 & 5.03 & BCS & $798_{-44}^{+53}$ & 255 \\
\hline A1930 & 218.1200 & 31.6330 & 0.1308 & 1.15 & BCS & $577_{-54}^{+75}$ & 76 \\
\hline A1978 & 222.7750 & 14.6110 & 0.1459 & 1.29 & BCS & $404_{-56}^{+95}$ & 63 \\
\hline A2009 & 225.0850 & 21.3620 & 0.1522 & 2.60 & BCS & $715_{-46}^{+57}$ & 195 \\
\hline RXJ1504 & 226.0321 & -2.8050 & 0.2168 & 14.12 & REF & $779_{-75}^{+105}$ & 120 \\
\hline A2034 & 227.5450 & 33.5060 & 0.1132 & 1.92 & BCS & $942_{-53}^{+64}$ & 182 \\
\hline A 2050 & 229.0680 & 00.0890 & 0.1191 & 1.18 & BCS & $869_{-61}^{+77}$ & 106 \\
\hline A2055 & 229.6720 & 06.2110 & 0.1023 & 1.34 & BCS & $676_{-64}^{+90}$ & 230 \\
\hline A2069 & 231.0410 & 29.9210 & 0.1139 & 2.46 & BCS & $994_{-52}^{+61}$ & 441 \\
\hline A2111 & 234.9337 & 34.4156 & 0.2291 & 3.35 & BCS & $741_{-52}^{+65}$ & 208 \\
\hline A2187 & 246.0591 & 41.2383 & 0.1829 & 1.56 & eBCS & $631_{-59}^{+83}$ & 103 \\
\hline A2219 & 250.0892 & 46.7058 & 0.2257 & 6.10 & BCS & $1151_{-54}^{+63}$ & 461 \\
\hline Zw8197 & 259.5480 & 56.6710 & 0.1132 & 0.80 & BCS & $597_{-53}^{+73}$ & 76 \\
\hline A2259 & 260.0370 & 27.6702 & 0.1605 & 1.85 & BCS & $855_{-60}^{+76}$ & 165 \\
\hline RXJ1720 & 260.0370 & 26.6350 & 0.1604 & 4.47 & BCS & $860_{-35}^{+40}$ & 376 \\
\hline
\end{tabular}


Table 1

(Continued)

\begin{tabular}{|c|c|c|c|c|c|c|c|}
\hline \multirow[t]{2}{*}{ Name } & \multicolumn{2}{|c|}{ X-Ray Coordinates } & \multirow[t]{2}{*}{$z_{\odot}$} & \multirow{2}{*}{$\begin{array}{l}L_{X} / 10^{43} \\
\left(\mathrm{erg} \mathrm{s}^{-1}\right)\end{array}$} & \multirow[t]{2}{*}{ Catalog } & \multirow{2}{*}{$\begin{array}{c}\sigma_{p} \\
\left(\mathrm{~km} \mathrm{~s}^{-1}\right)\end{array}$} & \multirow[t]{2}{*}{$N_{m}$} \\
\hline & R.A. (J2000) & Decl. (J2000) & & & & & \\
\hline A2261 & 260.6129 & 32.1338 & 0.2242 & 5.55 & $\mathrm{BCS}$ & $780_{-60}^{+78}$ & 209 \\
\hline RXJ2129 & 322.4186 & 00.0973 & 0.2339 & 5.65 & $\mathrm{BCS}$ & $858_{-57}^{+71}$ & 325 \\
\hline A2396 & 328.9198 & 12.5336 & 0.1919 & 1.86 & eBCS & $935_{-70}^{+90}$ & 176 \\
\hline A2631 & 354.4206 & 00.2760 & 0.2765 & 4.15 & eBCS & $851_{-72}^{+96}$ & 173 \\
\hline A2645 & 355.3200 & -9.0275 & 0.2509 & 2.85 & REF & $549_{-55}^{+78}$ & 61 \\
\hline
\end{tabular}

Table 2

HeCS Redshifts and Membership Classification

\begin{tabular}{|c|c|c|c|c|c|c|}
\hline \multicolumn{2}{|c|}{ Coordinates } & \multirow{2}{*}{$\begin{array}{c}c z_{\odot} \\
\left(\mathrm{km} \mathrm{s}^{-1}\right)\end{array}$} & \multirow{2}{*}{$\begin{array}{c}\sigma_{c z} \\
\left(\mathrm{~km} \mathrm{~s}^{-1}\right)\end{array}$} & \multirow[t]{2}{*}{$R$} & \multirow[t]{2}{*}{ Flag } & \multirow[t]{2}{*}{ Member } \\
\hline R.A. (J2000) & Decl. (J2000) & & & & & \\
\hline 1:50:49.556 & 1:02:12.196 & 69153 & 35 & 10.56 & Q & 0 \\
\hline 1:50:50.707 & 1:04:06.564 & 113666 & 33 & 13.70 & Q & 0 \\
\hline 1:50:52.011 & $0: 52: 56.928$ & 122005 & 36 & 12.00 & $\mathrm{Q}$ & 0 \\
\hline 1:50:52.308 & 1:05:49.391 & 188459 & 23 & 13.49 & Q & 0 \\
\hline 1:50:52.987 & $0: 58: 50.880$ & 106822 & 48 & 08.77 & Q & 0 \\
\hline 1:50:53.390 & $0: 53: 45.420$ & 68681 & 38 & 11.53 & $\mathrm{Q}$ & 1 \\
\hline
\end{tabular}

(This table is available in its entirety in a machine-readable form in the online journal. A portion is shown here for guidance regarding its form and content.)

After processing and reducing the spectra, we used the IRAF package rvsao (Kurtz \& Mink 1998) to cross-correlate the spectra with galaxy templates assembled from previous Hectospec observations. During the pipeline processing, spectral fits are assigned a quality flag of "Q" for high-quality redshifts, "?" for marginal cases, and " $\mathrm{X}$ " for poor fits. Repeat observations of several targets with "?" flags show that these redshifts are generally reliable. We visually inspected all spectra to include "?" and "X" spectra that have redshifts secured by multiple lines (usually four or more). Repeat observations indicate that the redshift uncertainties from rvsao are reasonable: Geller et al. (2012) use 1468 unique pairs of repeat observations to estimate a mean internal error of $56 \mathrm{~km} \mathrm{~s}^{-1}$ for absorption-line objects and $21 \mathrm{~km} \mathrm{~s}^{-1}$ for emission-line objects (see also Fabricant et al. 2005).

Here we include new redshifts for 57 of the HeCS clusters. For the remaining cluster, RXJ1720+26, Owers et al. (2011) obtained redshifts from MMT/Hectospec and Keck/DEIMOS for a separate investigation of cool-core clusters. The data for RXJ1720+26 do not extend to the full field of Hectospec, but the spectra do extend to fainter apparent magnitudes than HeCS.

We observed clusters at $z>0.15$ with two configurations to obtain larger samples and to mitigate issues with fiber collisions. We observed clusters at $z=0.10-0.15$ with one Hectospec configuration. Table 2 lists the 22,680 unique redshifts obtained for HeCS. Columns 1 and 2 provide celestial coordinates, Column 3 is the heliocentric redshift $c z$, Column 4 is the uncertainty in $c z$, Column 5 is the cross-correlation score $R$, Column 6 is the quality flag, and Column 7 is a membership flag indicating the number of clusters for which this galaxy is classified as a member. In our analysis, we also use several thousand redshifts from SDSS (mostly foreground galaxies) and 132 redshifts around A2219 from Boschin et al. (2004). For convenience, Table 3 provides these redshifts for the 2621 galaxies classified as members. In total, we identify 10,145 galaxies as members of a HeCS cluster. There are 334 galaxies that are members of two HeCS clusters (i.e., the infall regions of a few clusters overlap slightly).
Table 3

HeCS Members from Literature Redshifts

\begin{tabular}{|c|c|c|c|c|c|}
\hline \multicolumn{2}{|c|}{ Coordinates } & \multirow{2}{*}{$\begin{array}{c}c z_{\odot} \\
\left(\mathrm{km} \mathrm{s}^{-1}\right)\end{array}$} & \multirow{2}{*}{$\begin{array}{c}\sigma_{c z} \\
\left(\mathrm{~km} \mathrm{~s}^{-1}\right)\end{array}$} & \multirow[t]{2}{*}{ Reference } & \multirow[t]{2}{*}{ Member } \\
\hline R.A. (J2000) & Decl. (J2000) & & & & \\
\hline 1:52:17.93 & 01:04:57.65 & 67795 & 51 & 1 & 1 \\
\hline $1: 52: 50.47$ & $01: 12: 45.11$ & 68206 & 48 & 1 & 1 \\
\hline 7:56:35.16 & 54:04:28.60 & 30795 & 42 & 1 & 1 \\
\hline 7:58:18.82 & 53:57:37.69 & 30630 & 42 & 1 & 1 \\
\hline $7: 58: 44.16$ & 54:00:46.55 & 31295 & 39 & 1 & 1 \\
\hline
\end{tabular}

References. (1) SDSS; (2) Boschin et al. 2004.

(This table is available in its entirety in a machine-readable form in the online journal. A portion is shown here for guidance regarding its form and content.)

\section{RESULTS}

\subsection{Ubiquity of Infall Patterns Around Clusters}

We first search for well-defined infall patterns around X-ray clusters. Analogous to CIRS, we plot the rest frame line-of-sight velocity relative to the cluster center as a function of projected radius in Figure 2. All $58 \mathrm{HeCS}$ systems have "clean" infall patterns; that is, there is little ambiguity in the location of the caustics or limits of the pattern in redshift. All clusters contain a large number of members at the cluster redshift extending out to several Mpc from the cluster center.

Figure 1 shows the X-ray luminosities of the HeCS clusters compared to HIFluGCS (Reiprich \& Böhringer 2002) and CIRS. Due to the larger survey volume of HeCS, it includes many more systems with $L_{X} \gtrsim 10^{44} h^{-2} \mathrm{erg} \mathrm{s}^{-1}$ than the CIRS sample. There is a substantial overlap at intermediate $L_{X}$, especially at $z=0.10-0.15$. Table 1 lists the clusters in the HeCS sample, their X-ray positions and luminosities, their central redshifts, and rest-frame velocity dispersions. Velocity dispersions are measured for galaxies within $r_{200}$ as determined from the caustic mass profiles. The radius $r_{\Delta}$ is the radius within which the enclosed average mass density is $\Delta \rho_{c}$ (where $\rho_{c}$ is the critical density) by computing the enclosed density profile $\left(\rho(<r)=3 M(<r) / 4 \pi r^{3}\right) ; r_{200}$ is the radius which satisfies $\rho\left(<r_{200}\right)=200 \rho_{c}$.

Figure 2 shows the infall patterns and caustics for the HeCS clusters. The contrast in phase space density between cluster members and foreground/background galaxies is striking.

The clusters are ordered by decreasing X-ray luminosity. Velocity dispersions of clusters can be inferred visually from the spread in velocities at small radius. One immediate conclusion is that velocity dispersion and X-ray luminosity are not perfectly correlated: clusters such as RXJ1504 and A689 have large $\mathrm{X}$-ray luminosities, but their velocity dispersions are smaller than many clusters with comparable X-ray luminosity. Cooling cores of varying X-ray luminosity contribute to scatter in the $L_{X}-\sigma_{p}$ relation (e.g., Zhang et al. 2011). 


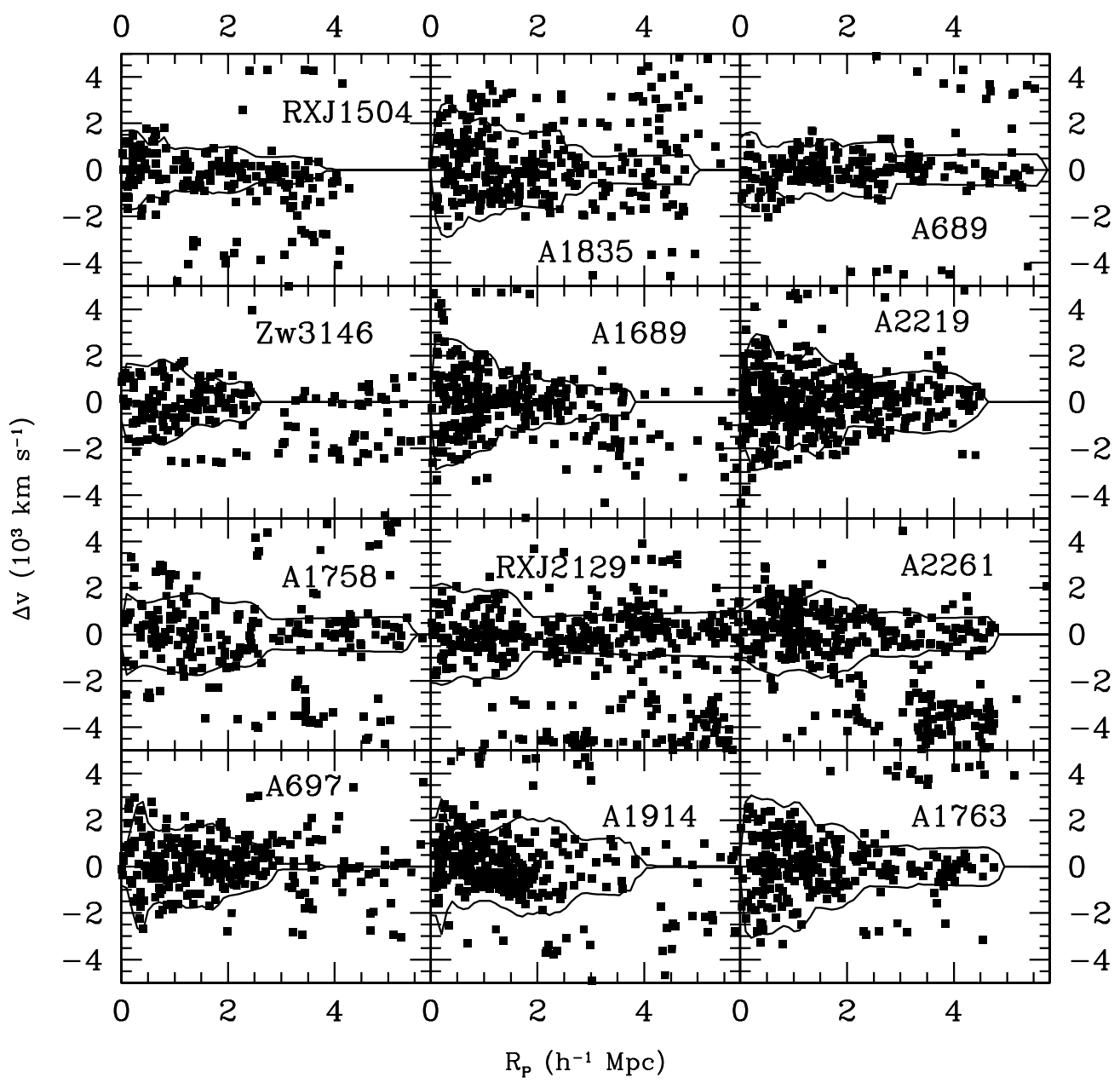

Figure 2. Redshift (rest-frame clustrocentric velocity) vs. projected radius for galaxies around HeCS clusters. The caustic pattern is evident as the trumpet-shaped regions with high density. The solid lines indicate our estimate of the location of the caustics in each cluster. Clusters are ordered left-to-right and top-to-bottom by decreasing X-ray luminosity.

(An extended version of this figure is available in the online journal.)

\subsection{Caustics and Mass Profiles}

We calculate the shapes of the caustics with the technique described in D99 using a smoothing parameter of $q=25$. The smoothing parameter $q$ is the scaling between the velocity smoothing and the radial smoothing in the adaptive kernel estimate of the underlying phase space distribution. Previous investigations show that the mass profiles are insensitive to changes of a factor of two in the smoothing parameter (Geller et al. 1999; Rines et al. 2000, 2002).

The technique of D99 uses the redshifts and coordinates of the galaxies to determine a hierarchical center based on a binary tree analysis. Analysis of 3000 simulated clusters indicates that the binary tree analysis recovers the input cluster center to within $\sim 300 \mathrm{~h}^{-1} \mathrm{kpc}$ for $95 \%$ of simulated clusters (Serra et al. 2011). For the HeCS clusters, the hierarchical centers are located within $300 h^{-1} \mathrm{kpc}$ of the X-ray coordinates for all but four clusters ( $7 \%$ of the sample, consistent with the $5 \%$ of simulated clusters with offsets this large). We discuss these four clusters and some other individual cases in Section 3.6. For one cluster (A2261), we use the slightly different algorithm for cutting the binary tree described by Serra et al. (2011) to determine the center.

Figure 2 shows the phase-space diagrams (rest-frame velocity versus projected radius) and the caustics. The D99 algorithm we use to identify the caustics generally agrees with the lines one would draw based on a visual impression. This consistency suggests that systematic uncertainties in the caustic technique are dominated by projection effects rather than the details of the algorithm (see Serra et al. 2011).

Figure 3 shows the associated caustic mass profiles. In redshift space, a cluster of galaxies appears as trumpet-shaped pattern (Regös \& Geller 1989; van Haarlem \& van de Weygaert 1993). DG and D99 demonstrated that for clusters forming hierarchically, the boundaries of this sharply defined pattern (termed caustics) in redshift space (phase space) can be identified with the escape velocity from the cluster. This identification provides a route to estimation of the cluster mass profile assuming spherical symmetry. This mass estimation method is called the caustic method.

The amplitude of the caustics $A(r)$ is half the distance between the boundaries of the cluster in redshift space. With the assumption of spherical symmetry the gravitational potential $\phi(r)$ and the caustic amplitude $A(r)$ are related by

$$
A^{2}(r)=-2 \phi(r) \frac{1-\beta(r)}{3-2 \beta(r)},
$$

where $\beta(r)$ is the anisotropy parameter, $\beta(r)=1-$ $\sigma_{\theta}^{2}(r) /\left[2 \sigma_{r}^{2}(r)\right]$, where $\sigma_{\theta}$ and $\sigma_{r}$ are, respectively, the tangential and radial velocity dispersions. 


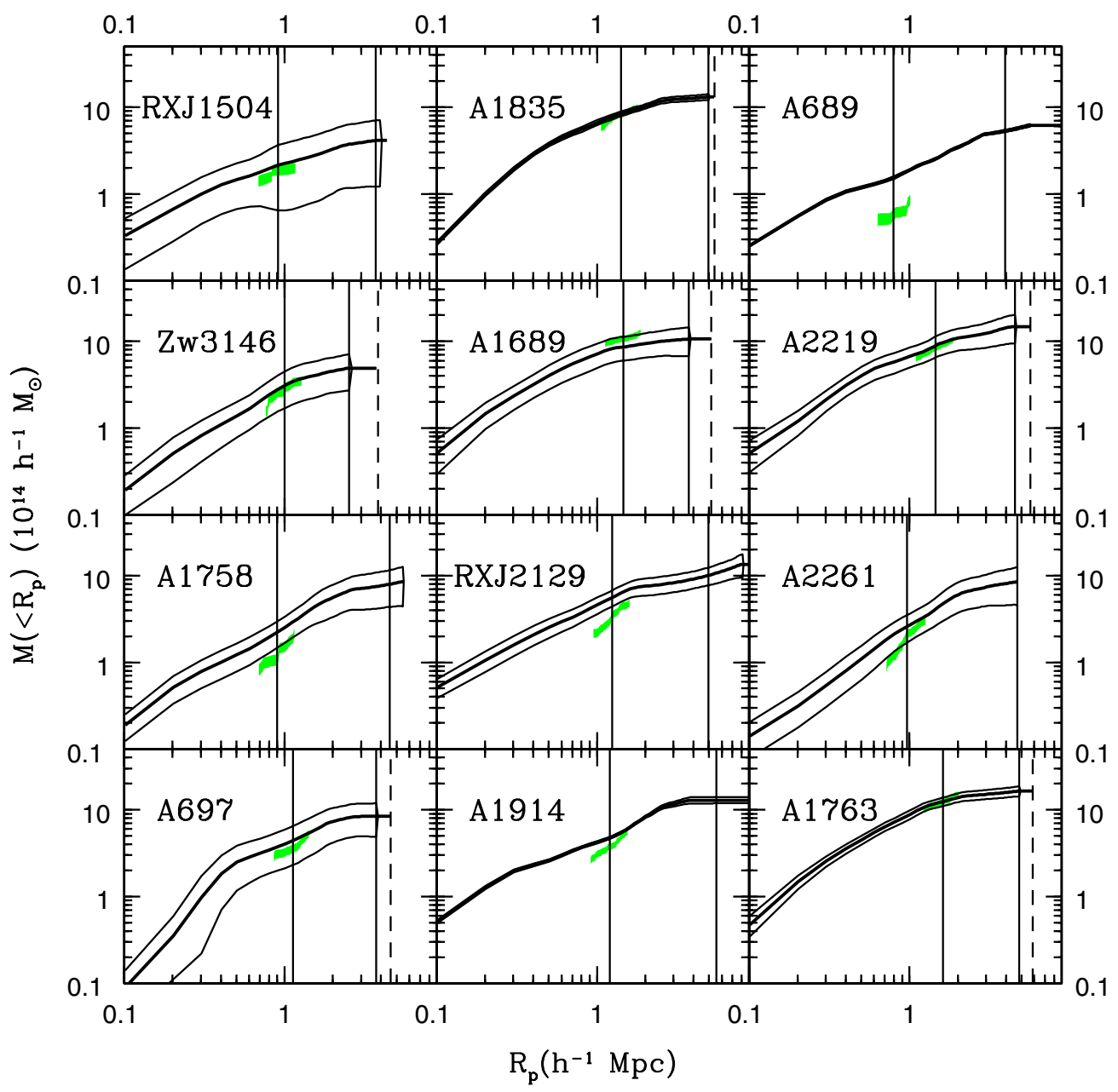

Figure 3. Caustic mass profiles for the HeCS clusters. The thick solid lines show the caustic mass profiles and the thin lines show the $1 \sigma$ uncertainties in the mass profiles. The inner vertical solid line in each panel shows the radius $r_{200}$. The next vertical line shows the smaller of $r_{5.6}$ (the limit of bound structure) and $r_{\text {max }}$ (the maximum radius where the caustics are detected). For clusters with $r_{\max }<r_{5.6}$, a dashed vertical line shows a lower limit on $r_{5.6}$ assuming no mass is present outside $r_{\max }$. Green shaded regions indicate the virial mass profile in the range (0.75-1.3) $r_{200}$ (approximately from $r_{500}$ to $r_{100}$ ).

(An extended, color version of this figure is available in the online journal.)

DG show that the mass of a spherical shell within the infall region is the integral of the square of the caustic amplitude $A(r)$ :

$$
G M(<r)-G M\left(<r_{0}\right)=\mathcal{F}_{\beta} \int_{r_{0}}^{r} A^{2}(x) d x
$$

where $\mathcal{F}_{\beta} \simeq 0.5$ is a filling factor with a value estimated from numerical simulations. We approximate $\mathcal{F}_{\beta}$ as a constant; variations in $\mathcal{F}_{\beta}$ with radius lead to some systematic uncertainty in the mass profile we derive from the caustic technique. In particular, the caustic mass profile assuming constant $\mathcal{F}_{\beta}$ somewhat overestimates the true mass profile within $\sim 0.5 h^{-1} \mathrm{Mpc}$ in simulated clusters (Serra et al. 2011). We include these issues in our assessment of the intrinsic uncertainties and biases in the technique (Serra et al. 2011).

Some investigators have experimented with a modified caustic technique utilizing a radially dependent $\mathcal{F}_{\beta}(r)$ tailored to match simulated clusters (e.g., Biviano \& Girardi 2003; Lemze et al. 2009). Because one goal of measuring mass profiles with the caustics is to test the predicted mass profiles from simulations, our approach is to assume a constant $\mathcal{F}_{\beta}$ rather than impose a functional form for $\mathcal{F}_{\beta}(r)$.

Note that the caustics extend to different radii for different clusters. These differences result in part from the varying physical size subtended by the Hectospec field at different redshifts.

D99 and Serra et al. (2011) show that the appearance of the caustics depends strongly on the line of sight; projection effects can therefore account for most of the differences in profile shape in Figure 3 without invoking non-homology among clusters.

We use the caustics to determine cluster membership. Here, the term "cluster member" refers to galaxies both in the virial region and in the infall region. Figure 2 shows that the caustics effectively separate cluster members from background and foreground galaxies. Some interlopers unavoidably lie within the caustics (e.g., Serra et al. 2011). Serra \& Diaferio (2012) applied the caustic technique to 3000 clusters extracted from $\mathrm{N}$-body simulations. The technique identifies $95 \% \pm 3 \%$ of the true cluster members within $3 r_{200}$. Only $2 \%$ of the galaxies inside the caustics and projected within $r_{200}$ are interlopers; the fraction of interlopers reaches $8 \%$ at $3 r_{200}$.

\subsection{Comparison to Virial Mass Estimates}

Zwicky $(1933,1937)$ first used the virial theorem to estimate the mass of the Coma cluster. With some modifications, notably a correction term for the surface pressure (The \& White 1986), the virial theorem remains in wide use (e.g., Girardi et al. 1998 
and references therein). Jeans analysis incorporates the radial dependence of the projected velocity dispersion (e.g., Carlberg et al. 1997; van der Marel et al. 2000; Biviano \& Girardi 2003, and references therein) and obviates the need for a surface term. Estimates of the mass profiles are complicated by the degeneracy between the mass and the velocity anisotropy (e.g., Mamon et al. 2013 and references therein).

Virial mass estimators rely on the assumption that galaxy orbits are in equilibrium, an assumption that is certainly violated in the infall region and probably also in the inner regions of clusters with significant substructure. Nevertheless, we apply the virial mass estimator to the HeCS clusters to check our caustic mass estimates. We must define a radius of virialization within which the galaxies are relaxed. We use $r_{200}$ as defined from the caustic mass profile (Table 4) and include only galaxies within the caustics. We thus assume that the caustics provide a good division between cluster galaxies and interlopers (see Figure 2).

We calculate the virial mass according to

$$
M_{\mathrm{vir}}=\frac{3 \pi}{2} \frac{\sigma_{p}^{2} R_{\mathrm{PV}}}{G},
$$

where $R_{\mathrm{PV}}=2 N(N-1) / \sum_{i, j>i} R_{i j}^{-1}$ is the projected virial radius and $\sigma_{p}^{2}=\sum_{i}\left[\left(v_{i}-\bar{v}\right) /(1+\bar{z})\right]^{2} /(N-1)$. If the system does not lie entirely within $r_{200}$, a surface pressure term $3 P V$ should be added to the usual virial theorem so that $2 T+U=$ $3 P V$. The virial mass is then an overestimate of the mass within $r_{200}$ by the fractional amount

$$
C=4 \pi r_{200}^{3} \frac{\rho\left(r_{200}\right)}{\int_{0}^{r_{200}} 4 \pi r^{2} \rho d r}\left[\frac{\sigma_{r}\left(r_{200}\right)}{\sigma\left(<r_{200}\right)}\right]^{2},
$$

where $\sigma_{r}\left(r_{200}\right)$ is the radial velocity dispersion at $r_{200}$ and $\sigma\left(<r_{200}\right)$ is the enclosed total velocity dispersion within $r_{200}$ (e.g., Girardi et al. 1998). In the limiting cases of circular, isotropic, and radial orbits, the maximum value of the term involving the velocity dispersion is $0,1 / 3$, and 1 , respectively. We estimate the uncertainties using the limiting fractional uncertainties $\pi^{-1}(2 \ln N)^{1 / 2} N^{-1 / 2}$. These uncertainties do not include systematic uncertainties due to membership determination. Table 4 lists the virial and caustic mass estimates at the radius $r_{200}$ determined from the caustic mass profile.

Figure 4 compares the virial and caustic mass estimates at $r_{200}$. The mean ratios of these estimates are $M_{c} / M_{v}=1.12 \pm 0.04$. The caustic mass estimates are slightly larger than virial mass estimates. Including a correction factor $C \approx 0.1-0.2 M_{\text {vir }}$, consistent with the best-fit NFW profiles (see also Carlberg et al. 1997; Girardi et al. 1998; Koranyi \& Geller 2000; Rines et al. 2003; CIRS), would lead to a larger difference. For the CIRS clusters, this ratio is slightly below unity. When we combine the CIRS and HeCS cluster samples (130 total), we obtain an average of $M_{c} / M_{v}=1.011_{-0.031}^{+0.033}$. These results indicate that the caustic mass profile and the virial theorem yields similar masses on the scale of the virial radius (approximately $r_{200}$ ).

Figure 3 compares the mass profiles estimated from the caustics and the virial theorem. The virial mass profiles are simply the virial mass estimator applied to all galaxies (inside the caustics) within that projected radius. Because the virial theorem only applies in regions where the galaxies are in equilibrium, we only display the virial mass profiles in the range $(0.75-1.3) r_{200}$ (corresponding roughly to $r_{500}$ to $r_{100}$ ). The virial and caustic mass profiles generally agree. That is, near the virial radius, the caustic mass profiles do not appear to consistently

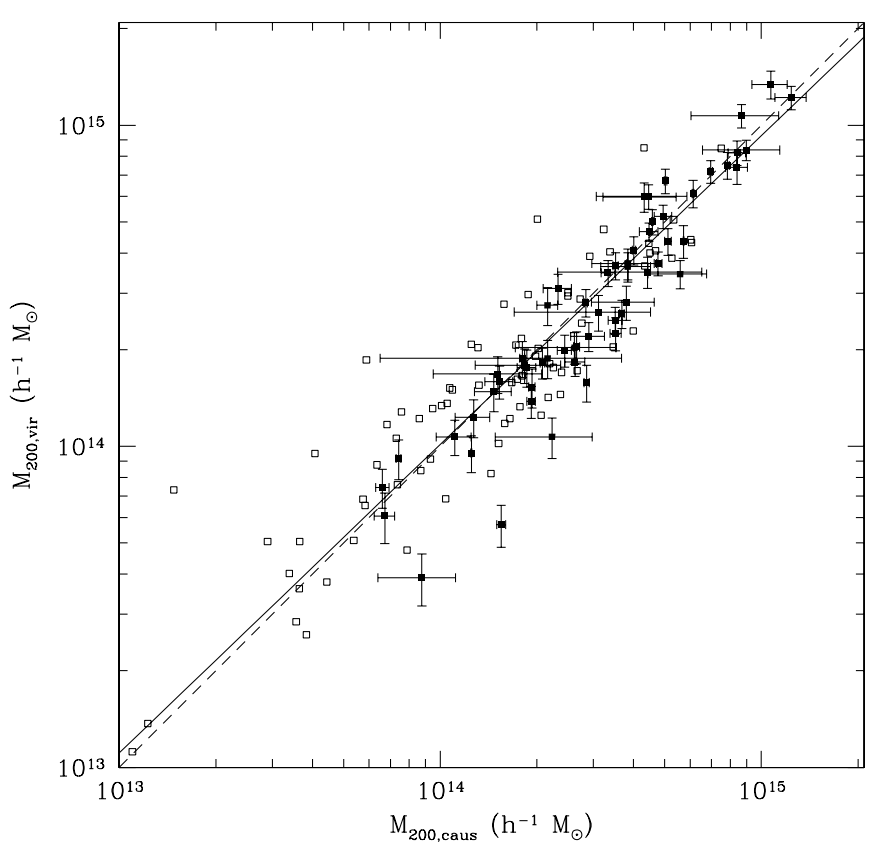

Figure 4. Caustic masses at $r_{200}$ (determined from the caustic mass profile) compared to virial masses at the same radius. Solid squares show HeCS clusters and open squares show clusters from CIRS. Error bars show $1 \sigma$ uncertainties and the dashed line has slope unity. The solid line is the bisector of the ordinary least-squares fits.

overestimate or underestimate the mass relative to the virial mass profiles. This result supports our use of caustic mass profiles as a tracer of the total cluster mass profile.

\subsection{Virial Masses and Ultimate Halo Masses}

The caustic mass profiles allow direct estimates of the virial and turnaround radius in each cluster. For the virial radius, we estimate $r_{200}$. In our adopted cosmology, a system should be virialized inside the slightly larger radius $\sim r_{100} \approx 1.3 r_{200}$ (Eke et al. 1996). We use $r_{200}$ because it is more commonly used in the literature and thus allows easier comparison of results.

Nagamine \& Loeb (2003) show that particles within a radius enclosing an overdensity of $\delta_{c}=17.6$ (enclosed density $5.55 \rho_{\text {crit }}$ or $\left.\left(9 \pi^{2} / 16\right) \rho_{\text {crit }}\right)$ in the present epoch remain bound to the central halo in numerical simulations of the far-future evolution of large-scale structure in a $\Lambda \mathrm{CDM}$ universe. This criterion is further supported by simulations by other investigators (Busha et al. 2003, 2005; Dünner et al. 2006). To be precise, Dünner et al. (2006) show that about $10 \%$ of the particles within this radius eventually become unbound, but that more distant particles constituting $13 \%$ of the mass within $\delta_{c}=17.6$ are ultimately accreted by the halo; thus, the mass within $\delta_{c}=17.6$ is 1.03 times smaller than the mass of the halo when the scale factor is $a=100 .^{6}$ If the $w$ parameter in the equation of state of the dark energy $\left(P_{\Lambda}=w \rho_{\Lambda}\right)$ satisfies $w \geqslant-1$, the dark energy has little effect on the turnaround overdensity (Gramann $\&$ Suhhonenko 2002). Varying $\Omega_{m}$ in the range $0.02-1$ only changes the inferred value of $r_{\text {turn }}$ by $\pm 10 \%$; the uncertainties in $r_{\text {turn }}$ from the uncertainties in the mass profile are comparable or larger (D99; Rines et al. 2002). Busha et al. (2005) quantify the ultimate mass of dark matter halos in their simulations by the ratio $M_{5.6} / M_{200}$, i.e., the ratio of the ultimate mass to the

\footnotetext{
6 In previous work, we used the slightly more generous definition of the turnaround radius $r_{\text {turn }}$ determined from Equation (8) of Regös \& Geller (1989) assuming $\Omega_{m}=0.3$. For this value of $\Omega_{m}$, the enclosed density is $3.5 \rho_{c}$ at the turnaround radius, versus $5.55 \rho_{c}$ for $\delta_{c}=17.6$.
} 
Table 4

HeCS Characteristic Radii and Masses

\begin{tabular}{|c|c|c|c|c|c|c|c|c|}
\hline Cluster & $\begin{array}{c}r_{500} \\
(\mathrm{Mpc})\end{array}$ & $\begin{array}{c}r_{200} \\
(\mathrm{Mpc})\end{array}$ & $\begin{array}{c}r_{5.6} \\
(\mathrm{Mpc})\end{array}$ & $\begin{array}{c}r_{\max } \\
(\mathrm{Mpc})\end{array}$ & $\begin{array}{c}M_{200} \\
\left(10^{14} M_{\odot}\right)\end{array}$ & $\begin{array}{c}M_{\mathrm{vir}} \\
\left(10^{14} M_{\odot}\right)\end{array}$ & $\begin{array}{c}M_{5.6} \\
\left(10^{14} M_{\odot}\right)\end{array}$ & $M_{\max } / M_{200}$ \\
\hline A267 & 0.80 & 1.19 & 4.26 & 4.85 & $4.95 \pm 0.31$ & $5.20 \pm 0.44$ & $6.32 \pm 0.44$ & $1.28 \pm 0.12$ \\
\hline Zw1478 & 0.41 & 0.64 & 2.68 & 3.64 & $0.66 \pm 0.03$ & $0.74 \pm 0.10$ & $1.38 \pm 0.08$ & $2.09 \pm 0.16$ \\
\hline A646 & 0.67 & 0.98 & 4.24 & 10.00 & $2.44 \pm 0.12$ & $1.99 \pm 0.23$ & $5.58 \pm 0.34$ & $2.29 \pm 0.24$ \\
\hline A655 & 0.74 & 1.08 & 4.46 & 8.89 & $3.33 \pm 0.16$ & $3.47 \pm 0.32$ & $6.50 \pm 0.42$ & $1.95 \pm 0.26$ \\
\hline A667 & 0.73 & 1.02 & 4.09 & 7.68 & $2.86 \pm 0.05$ & $1.58 \pm 0.21$ & $5.09 \pm 0.12$ & $1.78 \pm 0.06$ \\
\hline A689 & 0.55 & 0.80 & 3.95 & 5.66 & $1.55 \pm 0.05$ & $0.57 \pm 0.08$ & $5.32 \pm 0.16$ & $3.43 \pm 0.21$ \\
\hline A697 & 0.76 & 1.13 & 4.60 & 3.74 & $4.42 \pm 2.10$ & $3.49 \pm 0.39$ & $8.40 \pm 3.48$ & $1.90 \pm 1.20$ \\
\hline A750 & 0.61 & 0.99 & 4.20 & 4.04 & $2.63 \pm 0.19$ & $1.83 \pm 0.18$ & $5.64 \pm 0.95$ & $2.14 \pm 0.39$ \\
\hline MS0906 & 0.41 & 0.81 & 3.59 & 3.94 & $1.47 \pm 0.19$ & $1.48 \pm 0.20$ & $3.58 \pm 0.55$ & $2.44 \pm 0.52$ \\
\hline A773 & 0.99 & 1.40 & 5.16 & 5.35 & $7.84 \pm 0.10$ & $7.50 \pm 0.72$ & $11.10 \pm 0.30$ & $1.42 \pm 0.04$ \\
\hline A795 & 0.70 & 1.10 & 4.44 & 4.14 & $3.52 \pm 0.07$ & $3.65 \pm 0.35$ & $6.47 \pm 0.15$ & $1.84 \pm 0.06$ \\
\hline Zw2701 & 0.57 & 0.86 & 3.19 & 3.23 & $1.83 \pm 0.54$ & $1.79 \pm 0.22$ & $2.61 \pm 0.96$ & $1.43 \pm 0.67$ \\
\hline A963 & 0.74 & 1.12 & 4.55 & 4.54 & $4.01 \pm 0.05$ & $4.08 \pm 0.41$ & $7.49 \pm 0.11$ & $1.87 \pm 0.04$ \\
\hline A980 & 0.80 & 1.18 & 4.27 & 3.64 & $4.46 \pm 1.40$ & $6.00 \pm 0.53$ & $5.86 \pm 1.89$ & $1.31 \pm 0.59$ \\
\hline Zw3146 & 0.60 & 1.00 & 3.83 & 2.52 & $3.11 \pm 1.41$ & $2.62 \pm 0.33$ & $4.91 \pm 2.20$ & $1.58 \pm 1.01$ \\
\hline A990 & 0.55 & 0.83 & 3.19 & 3.64 & $1.51 \pm 0.56$ & $1.68 \pm 0.22$ & $2.42 \pm 0.89$ & $1.60 \pm 0.84$ \\
\hline Zw3179 & 0.43 & 0.63 & 2.87 & 2.22 & $0.67 \pm 0.05$ & $0.61 \pm 0.11$ & $1.77 \pm 0.12$ & $2.62 \pm 0.26$ \\
\hline A1033 & 0.66 & 1.03 & 3.88 & 10.00 & $2.84 \pm 0.03$ & $2.80 \pm 0.28$ & $4.26 \pm 0.06$ & $1.50 \pm 0.03$ \\
\hline A1068 & 1.01 & 1.47 & 5.48 & 10.00 & $8.40 \pm 0.66$ & $7.40 \pm 0.85$ & $12.20 \pm 1.20$ & $1.45 \pm 0.19$ \\
\hline A1132 & 0.76 & 1.10 & 4.73 & 4.24 & $3.52 \pm 0.14$ & $2.25 \pm 0.27$ & $7.84 \pm 0.56$ & $2.23 \pm 0.18$ \\
\hline A1201 & 0.60 & 0.99 & 4.08 & 4.65 & $2.66 \pm 0.06$ & $2.04 \pm 0.23$ & $5.17 \pm 0.15$ & $1.94 \pm 0.08$ \\
\hline A1204 & 0.49 & 0.74 & 2.71 & 3.94 & $1.11 \pm 0.14$ & $1.07 \pm 0.13$ & $1.53 \pm 0.25$ & $1.38 \pm 0.32$ \\
\hline A1235 & 0.57 & 0.84 & 3.23 & 7.88 & $1.53 \pm 0.15$ & $1.59 \pm 0.19$ & $2.42 \pm 0.36$ & $1.58 \pm 0.28$ \\
\hline A1246 & 0.86 & 1.22 & 4.89 & 4.54 & $5.12 \pm 0.12$ & $4.35 \pm 0.41$ & $9.18 \pm 0.32$ & $1.79 \pm 0.08$ \\
\hline A 1302 & 0.59 & 0.93 & 3.90 & 2.83 & $2.08 \pm 0.03$ & $1.82 \pm 0.20$ & $4.30 \pm 0.12$ & $2.06 \pm 0.07$ \\
\hline A1361 & 0.55 & 0.78 & 3.73 & 10.00 & $1.25 \pm 0.00$ & $0.95 \pm 0.12$ & $3.75 \pm 0.02$ & $3.00 \pm 0.03$ \\
\hline A1366 & 0.59 & 0.90 & 4.37 & 10.00 & $1.92 \pm 0.06$ & $1.38 \pm 0.16$ & $6.05 \pm 0.27$ & $3.15 \pm 0.18$ \\
\hline A1413 & 0.88 & 1.29 & 5.10 & 10.00 & $5.72 \pm 0.02$ & $4.36 \pm 0.51$ & $9.88 \pm 0.04$ & $1.73 \pm 0.01$ \\
\hline A1423 & 0.73 & 1.09 & 4.36 & 4.75 & $3.68 \pm 0.06$ & $2.59 \pm 0.26$ & $6.66 \pm 0.13$ & $1.81 \pm 0.05$ \\
\hline A1437 & 1.08 & 1.59 & 5.53 & 10.00 & $10.70 \pm 1.35$ & $13.40 \pm 1.33$ & $12.50 \pm 1.55$ & $1.17 \pm 0.21$ \\
\hline A 1553 & 0.81 & 1.19 & 4.14 & 3.84 & $4.58 \pm 0.03$ & $5.01 \pm 0.45$ & $5.42 \pm 0.04$ & $1.18 \pm 0.01$ \\
\hline A 1682 & 0.88 & 1.28 & 4.45 & 5.25 & $6.13 \pm 0.04$ & $6.13 \pm 0.60$ & $7.16 \pm 0.05$ & $1.17 \pm 0.01$ \\
\hline A1689 & 1.01 & 1.46 & 5.15 & 3.74 & $8.68 \pm 2.64$ & $10.70 \pm 0.90$ & $10.60 \pm 3.90$ & $1.22 \pm 0.58$ \\
\hline A1758 & 0.57 & 0.90 & 4.53 & 5.46 & $2.23 \pm 0.75$ & $1.07 \pm 0.15$ & $7.96 \pm 3.59$ & $3.57 \pm 2.23$ \\
\hline A1763 & 1.10 & 1.62 & 5.86 & 4.85 & $12.40 \pm 1.39$ & $12.20 \pm 1.02$ & $16.50 \pm 2.29$ & $1.33 \pm 0.24$ \\
\hline A1835 & 0.95 & 1.41 & 5.40 & 4.95 & $8.41 \pm 0.53$ & $8.21 \pm 0.72$ & $13.10 \pm 1.06$ & $1.56 \pm 0.16$ \\
\hline A1902 & 0.49 & 0.95 & 3.69 & 2.52 & $2.33 \pm 0.23$ & $3.10 \pm 0.33$ & $3.80 \pm 0.48$ & $1.63 \pm 0.26$ \\
\hline A1918 & 0.60 & 0.90 & 3.68 & 10.00 & $1.93 \pm 0.04$ & $1.53 \pm 0.21$ & $3.69 \pm 0.17$ & $1.91 \pm 0.10$ \\
\hline A1914 & 0.82 & 1.20 & 5.54 & 10.00 & $4.77 \pm 0.13$ & $3.71 \pm 0.32$ & $12.90 \pm 1.07$ & $2.70 \pm 0.24$ \\
\hline A1930 & 0.60 & 0.89 & 3.47 & 4.24 & $1.86 \pm 0.12$ & $1.76 \pm 0.23$ & $3.08 \pm 0.27$ & $1.66 \pm 0.19$ \\
\hline A1978 & 0.41 & 0.69 & 3.25 & 3.23 & $0.88 \pm 0.24$ & $0.39 \pm 0.07$ & $2.56 \pm 0.76$ & $2.92 \pm 1.30$ \\
\hline A2009 & 0.70 & 1.09 & 4.49 & 3.33 & $3.51 \pm 0.17$ & $2.47 \pm 0.24$ & $6.79 \pm 0.56$ & $1.93 \pm 0.19$ \\
\hline RXJ1504 & 0.61 & 0.91 & 3.71 & 3.94 & $2.16 \pm 1.51$ & $1.88 \pm 0.26$ & $4.12 \pm 2.91$ & $1.91 \pm 1.90$ \\
\hline A2034 & 0.81 & 1.25 & 4.41 & 3.23 & $5.03 \pm 0.05$ & $6.71 \pm 0.58$ & $6.20 \pm 0.07$ & $1.23 \pm 0.02$ \\
\hline A2050 & 0.76 & 1.18 & 4.04 & 3.03 & $4.32 \pm 1.11$ & $5.98 \pm 0.62$ & $4.78 \pm 1.28$ & $1.11 \pm 0.41$ \\
\hline A2055 & 0.61 & 0.94 & 3.75 & 10.00 & $2.16 \pm 0.16$ & $2.75 \pm 0.37$ & $3.78 \pm 0.34$ & $1.75 \pm 0.27$ \\
\hline A2069 & 0.85 & 1.39 & 5.86 & 10.00 & $6.96 \pm 0.08$ & $7.18 \pm 0.58$ & $14.60 \pm 0.20$ & $2.10 \pm 0.04$ \\
\hline A2111 & 0.69 & 1.00 & 4.24 & 4.75 & $2.90 \pm 0.35$ & $2.20 \pm 0.23$ & $6.19 \pm 1.04$ & $2.13 \pm 0.47$ \\
\hline A 2187 & 0.52 & 0.77 & 3.08 & 4.24 & $1.27 \pm 0.16$ & $1.23 \pm 0.17$ & $2.26 \pm 0.30$ & $1.78 \pm 0.32$ \\
\hline A 2219 & 0.97 & 1.46 & 5.67 & 4.54 & $8.98 \pm 2.42$ & $8.37 \pm 0.62$ & $14.80 \pm 5.36$ & $1.65 \pm 0.74$ \\
\hline Zw8197 & 0.57 & 0.89 & 3.33 & 2.32 & $1.80 \pm 0.03$ & $1.88 \pm 0.24$ & $2.66 \pm 0.06$ & $1.48 \pm 0.04$ \\
\hline A2259 & 0.77 & 1.12 & 4.44 & 3.54 & $3.84 \pm 0.68$ & $3.63 \pm 0.38$ & $6.63 \pm 1.23$ & $1.73 \pm 0.44$ \\
\hline RXJ1720 & 0.79 & 1.18 & 4.29 & 2.32 & $4.47 \pm 0.30$ & $4.66 \pm 0.30$ & $6.01 \pm 0.52$ & $1.34 \pm 0.15$ \\
\hline A2261 & 0.57 & 0.97 & 4.72 & 4.75 & $2.62 \pm 0.91$ & $2.03 \pm 0.23$ & $8.54 \pm 3.97$ & $3.26 \pm 1.89$ \\
\hline RXJ2129 & 0.80 & 1.24 & 4.97 & 8.08 & $5.59 \pm 1.16$ & $3.44 \pm 0.34$ & $10.10 \pm 2.34$ & $1.81 \pm 0.89$ \\
\hline A2396 & 0.74 & 1.11 & 4.29 & 4.04 & $3.84 \pm 0.87$ & $3.71 \pm 0.41$ & $6.19 \pm 1.47$ & $1.61 \pm 0.53$ \\
\hline A2631 & 0.70 & 1.07 & 4.50 & 5.25 & $3.80 \pm 0.84$ & $2.81 \pm 0.34$ & $7.84 \pm 2.01$ & $2.06 \pm 0.77$ \\
\hline A2645 & 0.43 & 0.63 & 2.29 & 3.43 & $0.74 \pm 0.01$ & $0.92 \pm 0.13$ & $1.01 \pm 0.03$ & $1.36 \pm 0.04$ \\
\hline
\end{tabular}

present value of $M_{200}$. They find that the mass ratio follow a log-normal distribution with a peak at 2.2 and a dispersion of 0.38 (about $10 \%$ of halos occupy a high-end tail due to halos merging with larger halos).
Table 4 lists $r_{200}, r_{5.6}$, and the masses $M_{200}$ and $M_{5.6}$ enclosed within these radii. For some clusters, the maximum extent of the caustics $r_{\max }$ is smaller than $r_{5.6}$. For these clusters, $M_{5.6}$ and $r_{5.6}$ are minimal values assuming that there is no additional 


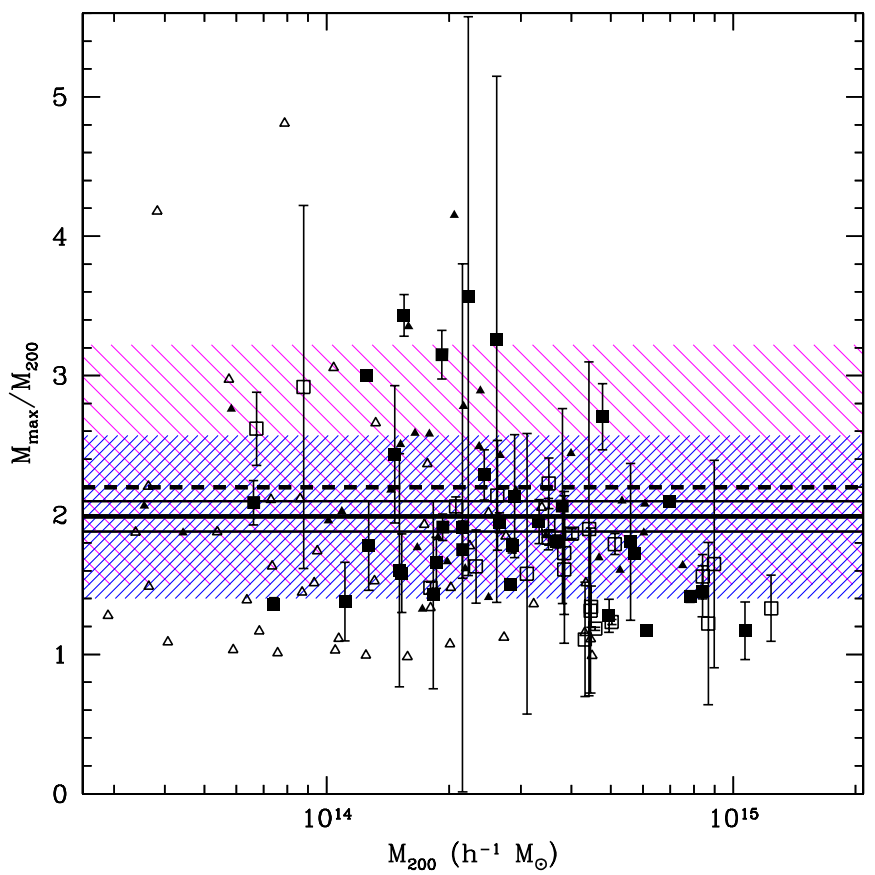

Figure 5. Ratio of mass $M_{\max }$ within the maximum radius of the caustics (or $r_{5.6}$ ) to the mass $M_{200}$ within $r_{200}$. Filled squares show clusters for which $r_{\max } \geqslant r_{5.6}$ and open squares show clusters with $r_{\max }<r_{5.6}$. The thick solid line shows the mean value of $M_{5.6} / M_{200}$ for clusters with $r_{\max } \geqslant r_{5.6}$. Thin solid lines show the uncertainty in this value. Triangles show CIRS clusters. The dashed line at $M_{5.6}=2.2 M_{200}$ is the ultimate mass of a halo in the far future (when the scale factor is $a=100$ ) compared to the present-day mass $M_{200}$ from the simulations of Busha et al. (2005). The distribution of $M(a=100) / M_{200}(a=1)$ in their simulations is well-described by a log-normal distribution with a dispersion shown by the magenta hatching (sloping down to the right). The dispersion in $\ln \left(M_{5.6} / M_{200}\right)$ for the HeCS clusters is shown by the dense blue hatching (sloping up to the right).

(A color version of this figure is available in the online journal.)

mass outside $r_{\max }$. The best estimate of the mass contained in infall regions clearly comes from those clusters for which $r_{\max } \geqslant r_{5.6}$. The average mass within $r_{5.6}$ for these clusters is $1.99 \pm 0.11$ times the virial mass $M_{200}$, in remarkable agreement with the prediction of $2.2 M_{200}$ (Busha et al. 2005). Further, the dispersion in $\ln M_{\max } / M_{200}$ is 0.30 (Figure 5), similar to the value of 0.38 for simulated clusters (Busha et al. 2005). The HeCS determination of $M_{5.6} / M_{200}$ demonstrates that clusters are still forming in the present epoch (this ratio is larger than unity). Further, the measurement is consistent with the CIRS estimate of $2.19 \pm 0.18$ (the CIRS estimate refers to $M_{3.5}$ rather than $M_{5.6}$; using $M_{5.6}$ for CIRS clusters would bring this value even closer to the HeCS estimate).

The remarkable agreement of the HeCS estimate of a cluster's ultimate halo mass with the prediction from simulations is a new test of $\Lambda \mathrm{CDM}$ structure formation theory.

Because estimating the ultimate halo mass of a cluster (assuming a $\Lambda \mathrm{CDM}$ model) requires determining the mass profile to a radius of $r_{5.6}$, the caustic technique is the only mass estimator that provides a direct probe of the ultimate halo mass. Weak lensing can detect shear signals at these radii, but the contribution of line-of-sight structure is difficult to separate from the lensing shear of the galaxies bound to the cluster.

The agreement between the estimates of $M_{5.6} / M_{200}$ and $M_{3.5} / M_{200}$ between the CIRS and HeCS samples suggests that the overall shapes of cluster mass profiles into their infall regions are not strongly dependent on cluster mass or redshift.

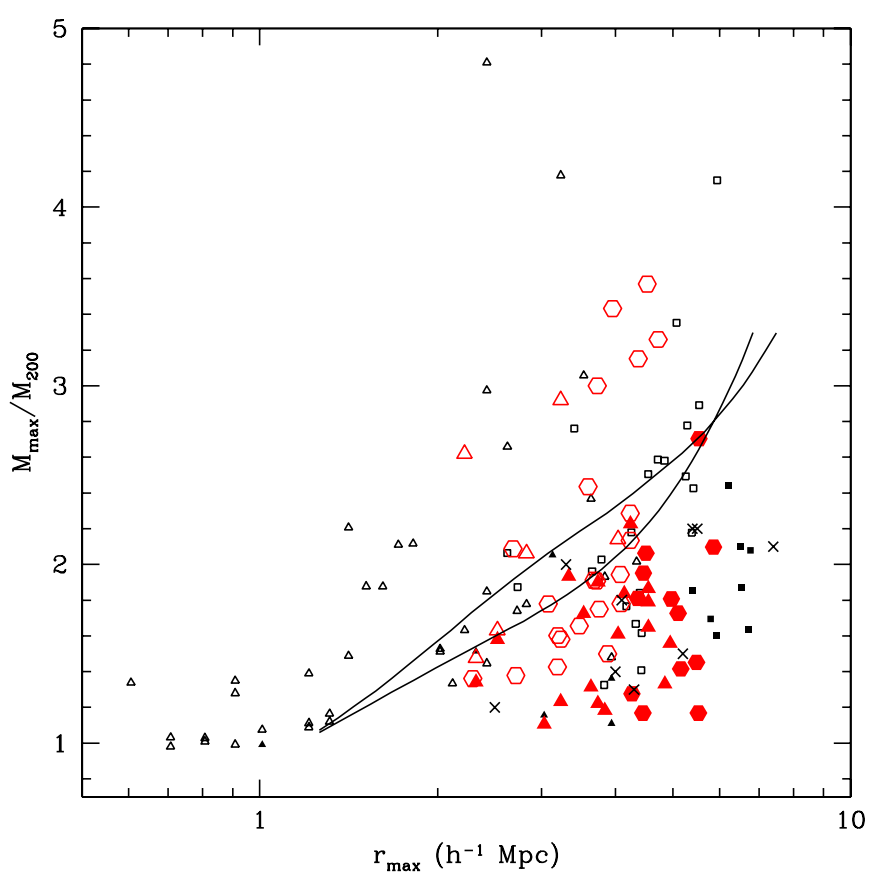

Figure 6. Ratio of mass $M_{\max }$ within the maximum radius of the caustics (or $r_{5.6}$ ) to $M_{200}$ vs. the maximum radius $r_{\max }$ of the caustics. Red hexagons are HeCS clusters with $M_{200}>3 \times 10^{14} h^{-1} M_{\odot}$; red triangles are HeCS with $M_{200}<3 \times 10^{14} h^{-1} M_{\odot}$. Filled points have $r_{\max } \geqslant r_{5.6}$. Black points are CIRS clusters. Filled squares show clusters for which $r_{\max } \geqslant r_{5.6}$ and open squares show clusters with $r_{\max }<r_{5.6}$. See Figure 5 for the typical uncertainties in $M_{\max } / M_{200}$. The lines show the mass profiles predicted by the simulations of Tinker et al. (2005).

(A color version of this figure is available in the online journal.)

In contrast, our mass profiles are perhaps in tension with the analysis of Tinker et al. (2005). Figure 6 compares our estimates of $M_{\max } / M_{200}$ as a function of $r_{\max }$ to the simulations of Tinker et al. (2005). Similar to CAIRNS and CIRS, the HeCS clusters tend to lie below the simulations, suggesting that either $M_{200}$ is overestimated or that $M_{\max }$ is often underestimated. However, the $\sim 50 \%$ offset suggested by Tinker et al. (2005) is difficult to reconcile with the good agreement between observed and simulated values of $M_{5.6} / M_{200}$ discussed above (see further discussion in Section 4).

One striking result of this analysis is that the caustic pattern is often visible beyond the radius $r_{5.6}$ that marks the maximum radius of galaxies that are gravitationally bound to the cluster. This result suggests that clusters may have strong dynamic effects on surrounding large-scale structure beyond the radius where galaxies will remain bound to the cluster in the far future.

\subsection{Cluster Scaling Relations}

Scaling relations between simple cluster observables and masses provide insight into the nature of cluster assembly and the properties of various cluster components. Establishing these relations for local clusters is critical for future studies of clusters in the distant universe with the goal of constraining dark energy (Majumdar \& Mohr 2004; Lin et al. 2004).

We apply the prescription of Danese et al. (1980) to determine the mean redshift $c z_{\odot}$ and projected velocity dispersion $\sigma_{p}$ of each cluster from all galaxies within the caustics. We calculate $\sigma_{p}$ using only the cluster members projected within $r_{200}$ estimated from the caustic mass profile. Note that our estimates of $r_{200}$ do not depend on $\sigma_{p}$.

Figure 7 shows the $M_{200}-\sigma_{p}$ relation. The tight relation indicates that the caustic masses are well correlated with 


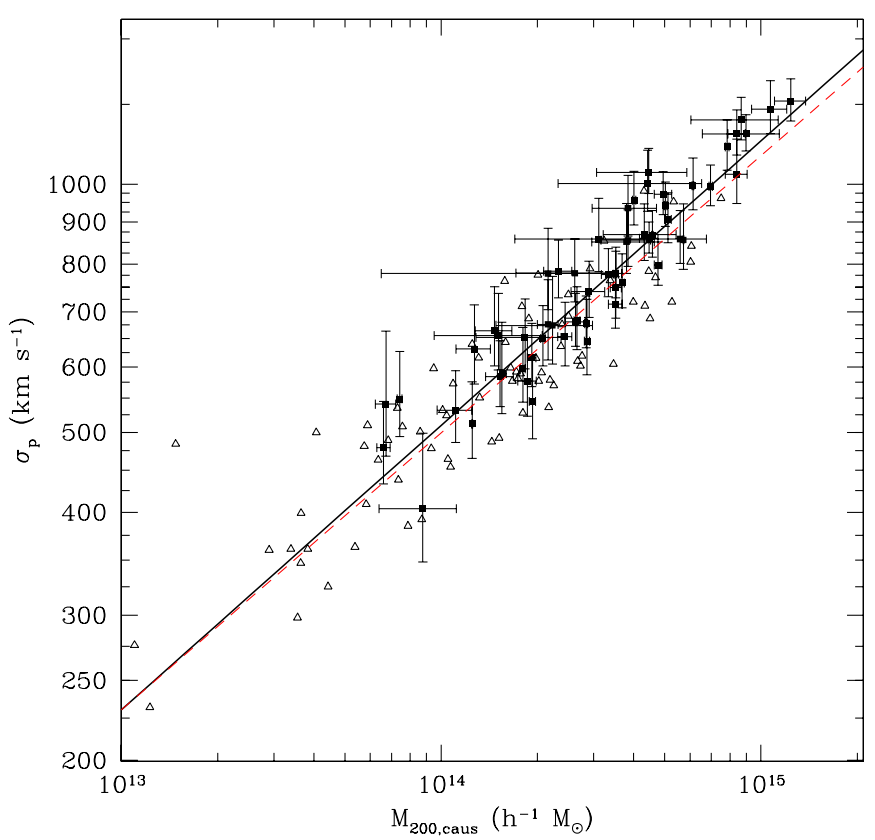

Figure 7. Caustic masses at $r_{200}$ compared to velocity dispersions within $r_{200}$. Squares and triangles show HeCS and CIRS clusters, respectively. The solid line is the bisector of the ordinary least-squares fits. The dashed line is the $\sigma_{p}-M_{200}$ relation of dark matter particles in cosmological simulations by Evrard et al. (2008).

(A color version of this figure is available in the online journal.)

velocity dispersion estimates. The good correlation is not surprising because both parameters depend on the galaxy velocity distribution. The best-fit slope is $M_{200} \propto \sigma_{p}^{2.90 \pm 0.15}$ with the uncertainty estimated from jackknife resampling. The dashed line in Figure 7 shows the $M_{200}-\sigma_{p}$ relation found by Evrard et al. (2008) for dark matter particles in simulated dark matter halos. Evrard et al. (2008) find that this relation is insensitive to variations in cosmological parameters or numerical resolution (above $10^{3}$ tracer particles). The excellent agreement between the observed CIRS and HeCS clusters and the virial scaling relations from simulated dark matter halos (slope $2.98 \pm 0.02$ ) suggests that the caustic technique yields accurate mass estimates.

Figure 8 compares the HeCS velocity dispersions to rest-frame X-ray luminosities in the ROSAT band. For reference, Figure 8 shows the best-fit $L_{X}-\sigma_{p}$ relation from Table C. 2 of Zhang et al. (2011) for $L_{X}$ measured in the ROSAT band including all emission within $r_{500}$ (i.e., before excluding emission from cooling cores). The HeCS clusters generally follow this relation, but there are also several outliers. Figure 8 labels several of these outliers. Two outliers, A689 and A1758, have problematic X-ray luminosities. Figure 8 shows that A689 lies close to the main locus of points when the X-ray luminosity is corrected for the central point source. Zhang et al. (2011) show that the $L_{X}-\sigma_{p}$ relation has smaller scatter when cool cores are excised from the $L_{X}$ measurements. Unfortunately, most HeCS clusters lack the high-resolution $X$-ray imaging required for this analysis. We defer a full analysis of the $L_{X}-\sigma_{p}$ relation to future work.

\subsection{Comments on Individual Clusters}

Clusters share many common features, but any large sample of clusters contains some complex systems. We comment on some

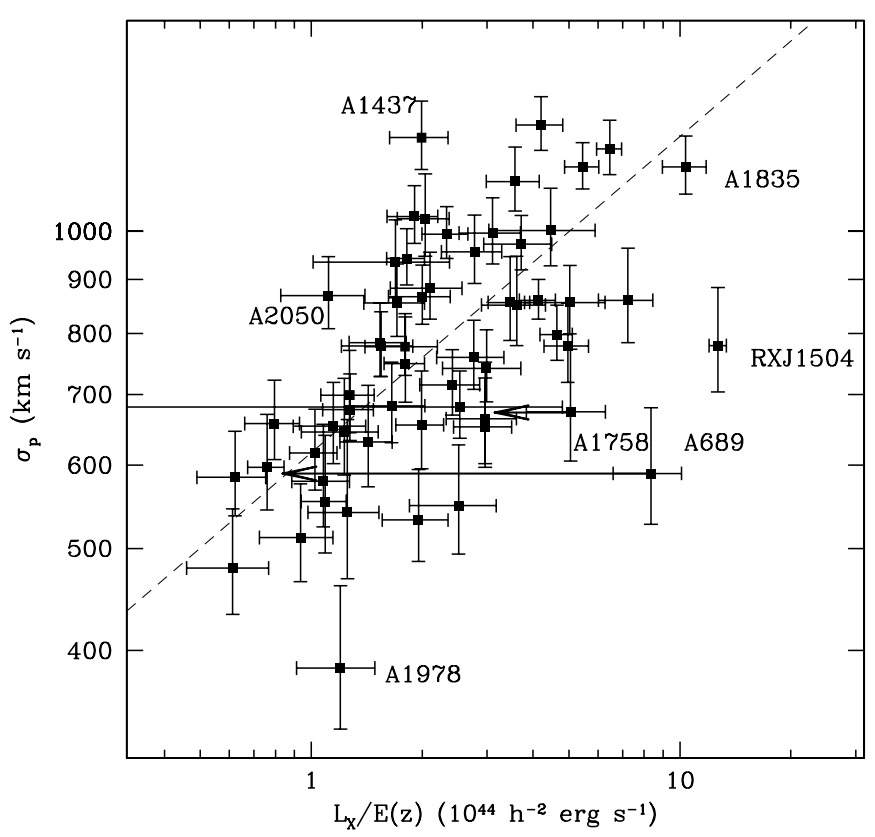

Figure 8. Rest-frame ROSAT X-ray luminosities compared to velocity dispersions within $r_{200}$. Several outliers are labeled, and arrows indicate corrected luminosities for A689 and A1758. The line shows the best-fit relation from Zhang et al. (2011) for $L_{X}$ in the ROSAT band and all emission within $r_{500}$ (i.e., before excluding emission from cooling cores).

of the most exceptional cases here. We present a comparison of caustic mass profiles and weak lensing mass profiles of several HeCS clusters in Geller et al. (2013).

A667.- The hierarchical center of A667 is located $307 h^{-1} \mathrm{kpc}$ north of the BCG. The X-ray center is close to the position of the BCG. The spatial distribution of cluster members shows significant substructure to the north. This substructure accounts for the offset in the centers.

A689.- This cluster was classified in BCS as a compact $\mathrm{X}$-ray source, suggesting possible contamination by a central point source (Ebeling et al. 1998). A recent Chandra observation confirms that most of the X-ray luminosity in BCS is due to a central point source identified as a BL Lac (Giles et al. 2012). Giles et al. (2012) estimate that the cluster luminosity in the BCS catalog is overestimated by about a factor of 10 . With the revised luminosity, A689 lies below the flux limit of our fluxlimited sample (Figure 1). The Hectospec redshifts confirm that the mass of A689 is significantly smaller than the masses of other clusters with similar (uncorrected) $L_{X}$. Figure 8 shows that A689 is not an outlier in the $L_{X}-\sigma_{p}$ diagram when using the corrected $L_{X}$. This cluster highlights the importance of highresolution X-ray observations in determining accurate X-ray luminosities.

MS0906/A750. - This pair of clusters is a curious system. MS0906+11 is an X-ray cluster at $z=0.1767$ detected in the Einstein Medium Sensitivity Slew Survey (Henry et al. 1992). A750 is a cluster at $z=0.1640$ located only $5^{\prime}\left(0.63 h^{-1} \mathrm{Mpc}\right)$ away from the X-ray center of MS0906 (see Figure 3.39 of Maughan et al. 2008). Carlberg et al. (1996, p. 37) noted that MS0906 "appears to be an indistinct binary in redshift space." With the denser sampling of HeCS, the infall patterns of the two clusters are separable. Figure 9 shows the caustics and mass profiles of these two clusters. The two clusters are separated by $3250 \mathrm{~km} \mathrm{~s}^{-1}$ (rest-frame), suggesting that they are not gravitationally bound. Note that the weak lensing map 


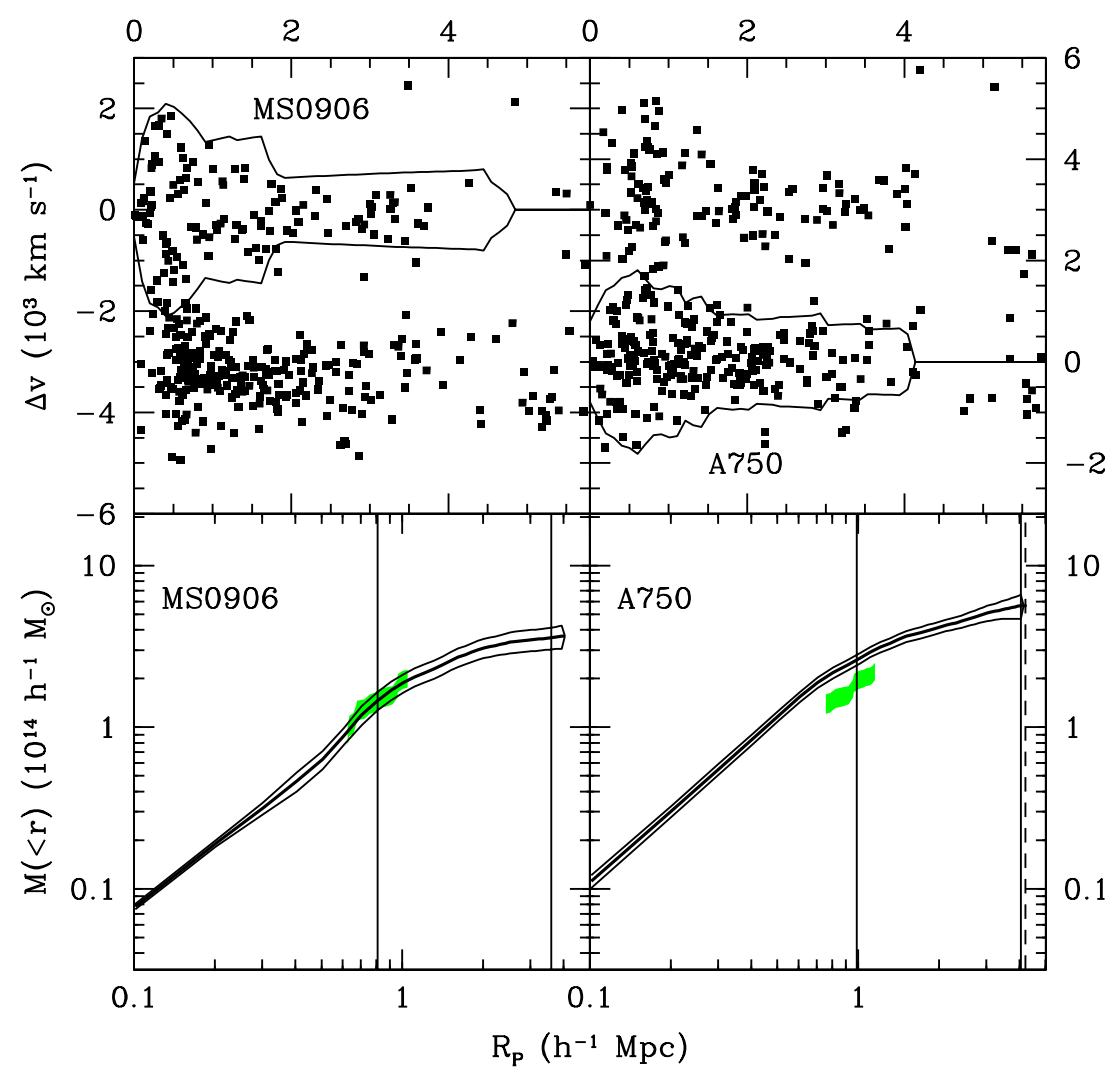

Figure 9. Top panels: redshift vs. projected radius for MS0906 (left) and A750 (right). The caustic pattern of A750 is much clearer when the plot is made with A750 at the center (cf. Figure 2). Bottom panels: mass profiles of MS0906 (left) and A750 (right).

(A color version of this figure is available in the online journal.)

of Okabe et al. (2010a) shows two distinct mass components centered approximately on MS0906 (component A750-C in their Figure 32) and A750 (component A750-NW1 in their Figure 32); MS0906 has a larger surface mass density and A750 has a larger luminosity density of red-sequence galaxies. The X-ray luminosity of MS0906 is much larger than that of A750 (Maughan et al. 2008). Our caustic mass profiles indicate that the two clusters have roughly equal mass. This complex system indicates that the relation between cluster mass and different observables is complicated. A more detailed analysis of this system is presented in Geller et al. (2013).

A773.-A773 contains a radio halo (Giovannini et al. 1999), a feature commonly associated with major mergers. Barrena et al. (2007) studied the dynamics of 100 cluster members and found evidence of complicated dynamics, including two velocity peaks separated by $\approx 2500 \mathrm{~km} \mathrm{~s}^{-1}$. Each velocity peak contains one of the two bright galaxies lying in the cluster center. A third galaxy (SDSS J091758.60+515104.6) is intermediate in brightness to the two bright galaxies in the core; this third galaxy is in the lower-velocity peak and is relatively isolated. The caustics contain both systems, although some galaxies in the high-velocity peak lie outside the caustics. Because the caustic method does not require equilibrium, the caustic method may be a more robust mass estimator for A773 than virial analysis. The hierarchical center has a redshift similar to the low-velocity peak, and the caustics at large radii are centered at the same redshift. Note that our estimate of the velocity dispersion $\left(1110_{-70}^{+86} \mathrm{~km} \mathrm{~s}^{-1}\right)$ is smaller than the global velocity dispersion $1394_{-68}^{+84} \mathrm{~km} \mathrm{~s}^{-1}$ of Barrena et al. (2007), although they find much smaller velocity dispersions of individual subclusters.
A1437. - This cluster was studied by Pimbblet et al. (2006). They measured a velocity dispersion of $1152_{-51}^{+59} \mathrm{~km} \mathrm{~s}^{-1}$, smaller than but consistent with our estimate $\left(\sigma_{p}=1233_{-81}^{+102} \mathrm{~km} \mathrm{~s}^{-1}\right)$. A1689.- This cluster is a famous strong-lensing cluster (e.g., Broadhurst et al. 2005). Łokas et al. (2006) studied the dynamics of A1689 from literature data and find significant lineof-sight substructure. The dynamics have also been studied by Lemze et al. (2009) using more extensive VLT/VIMOS spectroscopy obtained by Czoske (2004). Lemze et al. (2009) show that the mass profile determined with the caustic technique is consistent with mass profiles from gravitational lensing, X-ray data, and Jeans' analysis. Haines et al. (2010) use 1009 Hectospec redshifts (from an independent investigation) to study the properties of star-forming galaxies detected by Herschel. Note that Lemze et al. (2009) claim to detect the "edge" of A1689 by noting a sharp decrease in galaxy density at $R_{p} \approx 2.1 h^{-1} \mathrm{Mpc}$. Figure 2 shows several spectroscopically confirmed members beyond this radius, and we detect caustics extending to $R_{p}=3.7 h^{-1} \mathrm{Mpc}$.

A1758. - This system is a merger of multiple X-ray clusters at $z=0.28$. Several authors separate the cluster into A1758N and A1758S (e.g., Ebeling et al. 1998; David \& Kempner 2004). The combined flux of the two clusters (Böhringer et al. 2000) exceeds our flux limit; the individual fluxes lie below the flux limit. David \& Kempner (2004) showed that A1758N is itself a merger of two $7 \mathrm{keV}$ clusters and that A1758S shows evidence of a recent merger. Okabe \& Umetsu (2008) used Subaru to analyze the lensing properties of A1758N and A1758S and found confirming evidence that both components are undergoing mergers. Ragozzine et al. (2012) use a higher-resolution lensing analysis of $\mathrm{A} 1758 \mathrm{~N}$ and conclude that $\mathrm{A} 1758 \mathrm{~N}$ consists of 
two separate clusters A1758N:NW and A1758N:SE. Because A1758 is one of the highest-redshift clusters in HeCS, it is not sampled very deeply. A detailed dynamical analysis of this complex system would require additional data. The complex dynamics of the mergers in A1758 may contribute to its unusually high star formation rate (Haines et al. 2009). Boschin et al. (2012) study the dynamics of A1758N:NW and A1758N:SE using TNG spectroscopy. They find a much larger velocity dispersion $\left(\sigma_{p} \sim 1300 \mathrm{~km} \mathrm{~s}^{-1}\right)$ than we do, but they note that many high-velocity-offset galaxies might be members of subclusters.

A1902.-The hierarchical center is coincident with a close grouping of galaxies $531 h^{-1} \mathrm{kpc}$ E of the BCG. The close grouping is probably a merging event, leading to a very high binding energy that causes the hierarchical analysis to choose this grouping as the cluster center. The X-ray center is located close to the BCG, and the spatial distribution of cluster members shows many more members east of the BCG than west of the BCG. The BCG has some close companion galaxies that may have been missed by either SDSS photometry or by fiber collisions. In either case, the BCG companion galaxies would be omitted from the redshift catalog used for the hierarchical analysis; including some of these companions could conceivably relocate the hierarchical center close to the BCG.

A1914.-Although the X-ray contours of A1914 are fairly regular, it contains a large radio halo and significant $\mathrm{X}$-ray temperature anisotropy, two features consistent with a major merger (Govoni et al. 2004). Similarly, a weak lensing map by Okabe \& Umetsu (2008) shows significant substructure in both the lensing mass distribution and the luminosity distribution (which includes two bright galaxies close to but not coincident with the X-ray center). The HeCS data (Figure 2) show a clear caustic pattern but with an unusual shape: the median redshift of cluster members increases from the center to $\sim 0.5 h^{-1} \mathrm{Mpc}$, then decreases from this radius to $\sim 2.0 h^{-1} \mathrm{Mpc}$. Inspection of the spatial distribution of the cluster members reveals that a large group is located $\approx 1.5 h^{-1} \mathrm{Mpc}$ NW of the X-ray center. The group is offset by $\approx-1000 \mathrm{~km} \mathrm{~s}^{-1}$ from the redshift center of A1914 and has a velocity dispersion of $\sigma_{p} \sim 350 \mathrm{~km} \mathrm{~s}^{-1}$. This group largely explains the unusual appearance of the infall pattern in Figure 2. We defer a full dynamical analysis of this complex system to future work.

A1930.-The hierarchical center is located $385 h^{-1} \mathrm{kpc}$ from the X-ray center. The hierarchical center is 3.5 SSE of the BCG and $1^{\prime}$ from the second-ranked galaxy. The X-ray center is 2.5 WSW of the BCG. The BCG is located about 1'.5 NNE of the midpoint of the hierarchical center and the X-ray center.

RXJ1504-03.- This cluster is near the edge of the DR6 photometric footprint. Our target photometry is based on DR5, which did not contain the entire cluster. Part of the Hectospec field was contained in DR5 imaging that did not yield photometry. We used the SDSS Navigate tool to identify and include red and blue galaxies in our target list by inspecting the unprocessed SDSS imaging. A small portion of the Hectospec field was covered by neither SDSS photometry nor Atlas imaging. We added targets from POSSII plate scans in this region.

RXJ1504 is well known as a cooling-core cluster (Böhringer et al. 2005), and it is the most X-ray luminous cluster in HeCS. Vikhlinin et al (2009) estimate a large mass for RXJ1504 based on Chandra observations and the $Y_{X}$ estimator. However, Figures 2 and 8 show that its velocity dispersion is smaller than many clusters of comparable $X$-ray luminosity. This result suggests that X-ray mass estimates of RXJ1504 (including $Y_{X}$ ) may be biased due to the cooling core. Recently, Zhang et al. (2012) studied the dynamics of galaxies in RXJ1504 using VLT/ VIMOS spectroscopy. They identify 53 cluster members and compute a velocity dispersion of $\sigma_{p}=(1132 \pm 95) \mathrm{km} \mathrm{s}^{-1}$, a value significantly larger than ours $\left(\sigma_{p}=779_{-75}^{+105} \mathrm{~km} \mathrm{~s}^{-1}\right.$, Table 1). Comparing their Figure 7 to our Figure 2 indicates that their membership selection is more generous than our caustic selection; HeCS includes more redshifts (120 members versus 53 ) and covers a wider field-of-view than the spectra contained in Zhang et al. (2012). In addition, Zhang et al. (2012) identify several blue galaxies as cluster members: if these galaxies have a larger velocity dispersion than the red galaxies we target, this difference could partly explain the difference in measured velocity dispersions. However, analysis of blue galaxies in other HeCS clusters (Section 5.3) shows no significant color dependence of the velocity distribution of members identified with large redshift samples and the caustic technique.

A2055.- This cluster was studied by Pimbblet et al. (2006). They measured a velocity dispersion of $1046_{-65}^{+80} \mathrm{~km} \mathrm{~s}^{-1}$, significantly larger than our estimate $\left(\sigma_{p}=676_{-64}^{+90}\right)$. Inspection of the infall pattern shown in their Figure 5 reveals that they classify three galaxies with large velocity offsets as members; similar galaxies are classified as non-members by the caustic technique (Figure 2).

A2219.-The kinematics of this well-known lensing cluster (Smail et al. 1995) were studied by Boschin et al. (2004) using 132 redshifts within $5^{\prime}$ of the BCG. They find significant evidence of substructure and possible merging activity. We include their redshifts in our caustic analysis and we removed these galaxies from our Hectospec target list. A2219 contains a radio halo (Giovannini et al. 1999). Figure 2 shows that there are a few galaxies projected in front of A2219; while we classify these galaxies as foreground, Boschin et al. (2004) classify them as members. This membership difference probably accounts for most of the difference between their estimated velocity dispersion $\left(\sigma_{p}=1438_{-86}^{+109} \mathrm{~km} \mathrm{~s}^{-1}\right)$ and our estimate (Table 1). These foreground galaxies could also enhance the probability of observing strongly lensed background galaxies.

Zw8197.-The hierarchical center is located $8^{\prime}(667 \mathrm{kpc}) \mathrm{W}$ of the X-ray center. The X-ray center lies close to the BCG, but there are few/no galaxies around the BCG. The spatial distribution of cluster members is remarkably flat; the BCG lies in a sparse region of cluster members. The large magnitude gap between the BCG and other cluster members led Santos et al. (2007) to identify Zw8197 as a candidate fossil group.

A2261.-This system is near the edge of the DR6 photometric footprint. Although imaging is available through the SDSS Image List tool, photometry was not available in DR6 for much of the cluster (this situation can arise when a field is taken in poor seeing). We used the Guide Star Catalog to identify likely galaxies in the cluster region and the Image List tool to visually inspect these candidates. We experimented with using colors from the Guide Star Catalog photometry, but these identifications are less reliable than visual classification of the SDSS thumbnails. DR8 contains photometry for A2261. We used this photometry to select targets for an additional Hectospec pointing. We increased the sampling density and included blue galaxies to examine detailed issues in the strong and weak lensing determination of the cluster mass (Coe et al. 2012).

The D99 binary tree analysis we use for all other clusters locates the center of A2261 on a structure $6.3\left(\approx 1 h^{-1} \mathrm{Mpc}\right)$ and $400 \mathrm{~km} \mathrm{~s}^{-1}$ away from the BCG. This center lies atop a tight grouping $\left(30^{\prime \prime}\right)$ of four bright red cluster members (two 


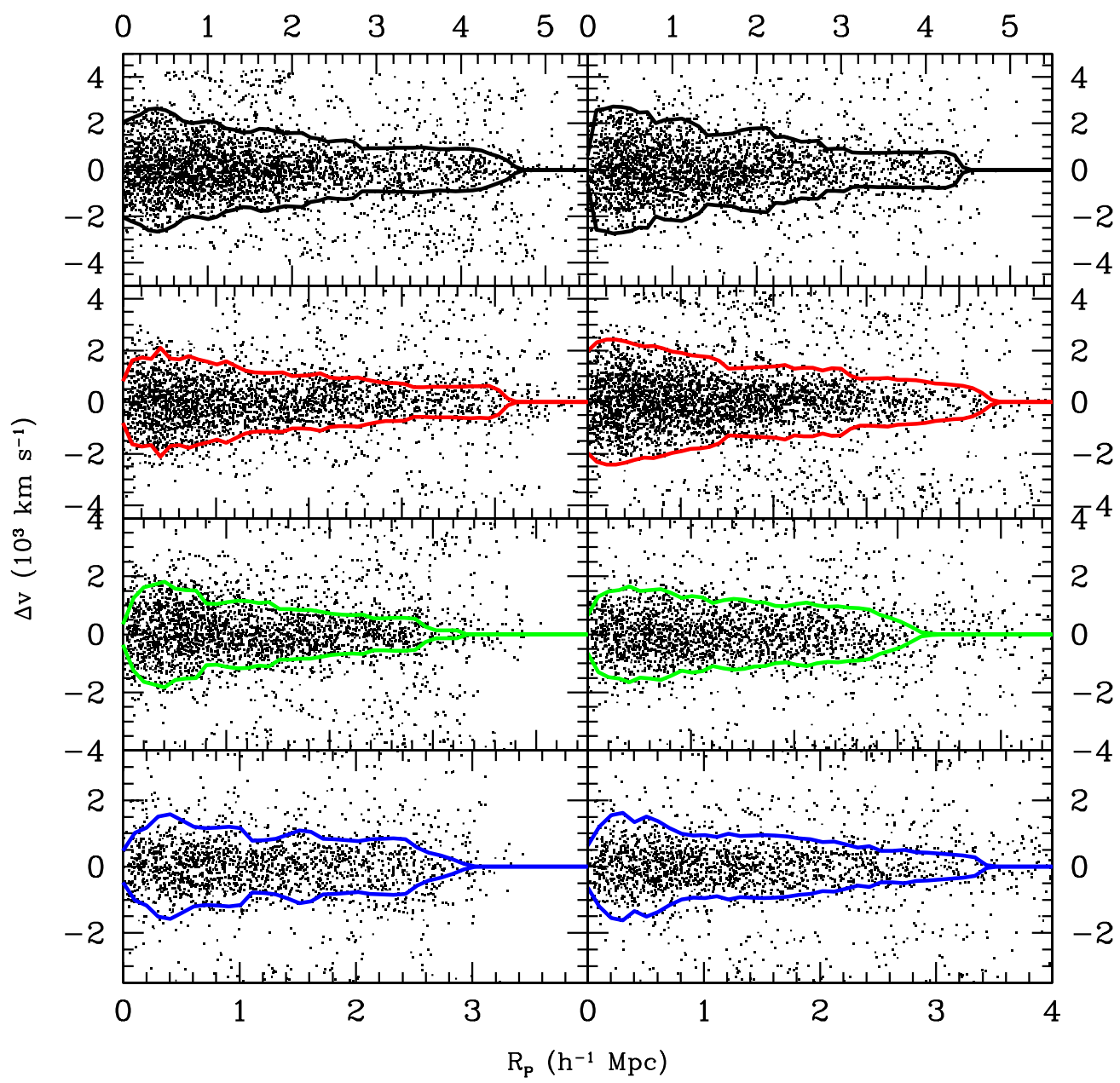

Figure 10. Redshift vs. projected radius in ensemble clusters. Left: quartiles of $L_{X}$; right: quartiles of $M_{200}$. Quartiles are shown from top to bottom by decreasing mass/luminosity. Solid lines indicate the positions of the caustics for the ensemble clusters.

(A color version of this figure is available in the online journal.)

with $L \sim 10 L_{*}$ ). However, the slightly different algorithm for cutting the binary tree described in Serra et al. (2011) yields the cluster center on the BCG. These two different results are a consequence of the complex dynamics of A2261. We adopt the center closest to the BCG. The caustic mass profile is insensitive to the adopted center.

Two teams have investigated the mass profile of A2261 using gravitational lensing. Okabe et al. (2010a) find that A2261 is "over-concentrated" relative to the expectations from numerical simulations. By contrast, Coe et al. (2012) use Hubble Space Telescope lensing data to measure a concentration closer to the expected value for a massive cluster. Coe et al. (2012) show that lensing estimates of $M_{200}$ can differ by $\sim 25 \%$ depending on the assumed cluster geometry (spherical versus triaxial). This flexibility allows the lensing mass to agree with various $\mathrm{X}$-ray hydrostatic mass estimates that differ by 35\% (and could be affected by non-thermal pressure support). However, both the $\mathrm{X}$-ray and lensing mass estimates are larger than our estimate of $M_{200}$ (see Figure 12 of Coe et al. 2012).

\section{ENSEMBLE CLUSTERS: THE CLUSTER MASS PROFILE}

The largest uncertainty in determining dynamical masses of clusters is the influence of projection effects. Stacking clusters together to create an ensemble cluster can significantly reduce this uncertainty (Serra et al. 2011). We create two sets of ensemble clusters using two methods of grouping the clusters. For all ensemble clusters, we eliminate A1758 and MS0906/A750 because they are double clusters (see Section 3.6).

First, we divide the clusters into quartiles of $M_{200}$ as determined from the caustic mass profile. We then stack the clusters in physical units (positions in $\mathrm{kpc}$, velocities in $\mathrm{km} \mathrm{s}^{-1}$ ). This method has the disadvantage that it relies on the parameters from the caustic mass profiles to assign the clusters into quartiles, so the resulting ensemble properties are not completely independent of the caustic mass profiles of the individual clusters.

Second, we divide the clusters into quartiles of X-ray luminosity from the original ROSAT catalogs. We then stack the clusters in physical units (positions in kpc, velocities in $\mathrm{km} \mathrm{s}^{-1}$ ). This approach avoids any use of the individual cluster caustic mass profiles in the stacking parameters.

Figure 10 shows the two sets of ensemble clusters. Both sets show a clear distinction between cluster members and field galaxies in all quartiles. Table 5 lists the median and ranges of the quartiles.

Figure 11 shows the density profiles of the ensemble clusters. The caustics trace the mass profiles across nearly four orders of magnitude in density. The blue dashed line shows an NFW profile with a concentration of $c=2.9$. The low-concentration 


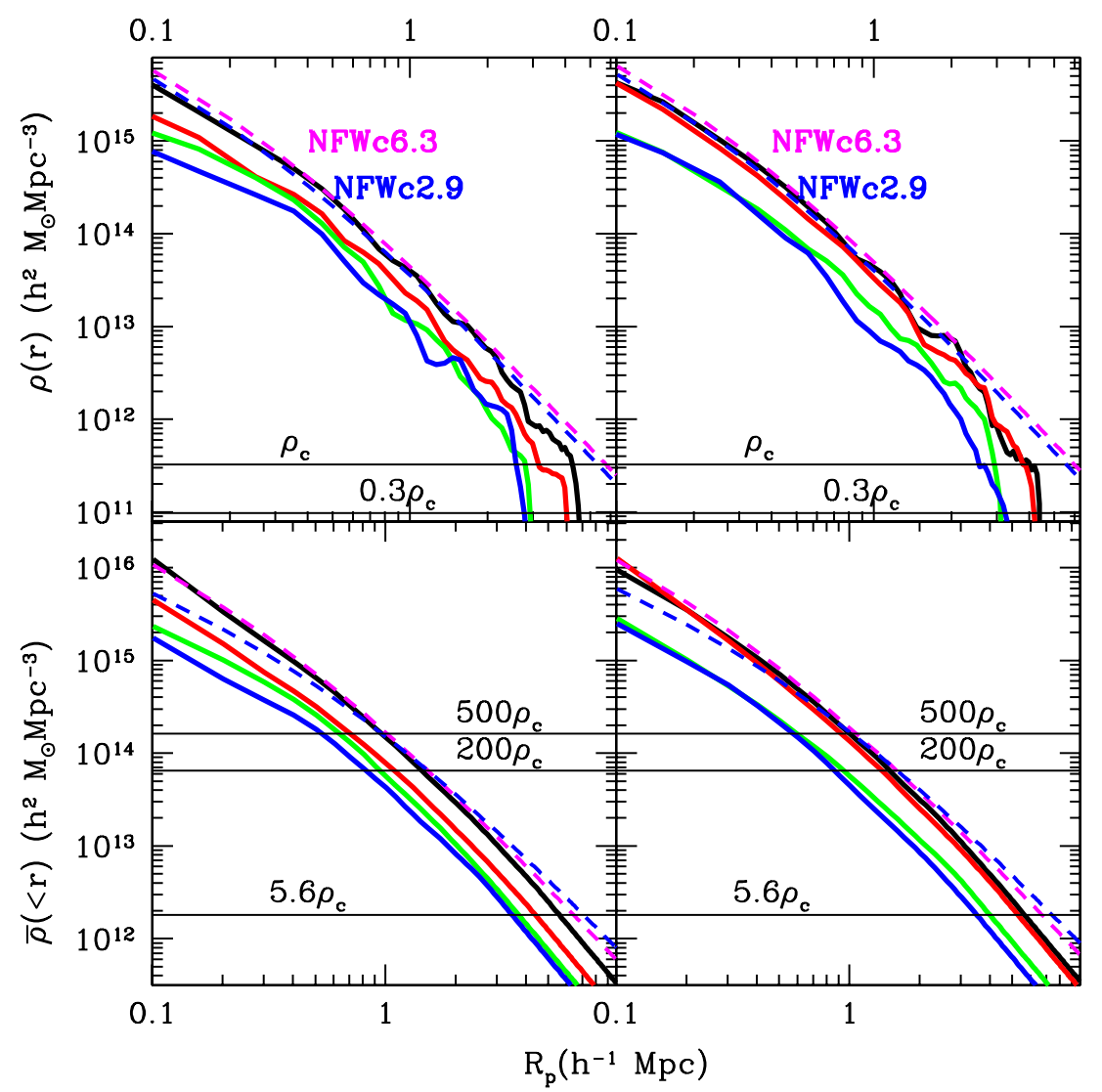

Figure 11. Density profiles of ensemble clusters. Black, red, green, and blue solid lines show decreasing quartiles of $L_{X}$ (left panels) or $M_{200}$ (right panels). Top panels: the magenta dashed line shows the NFW profiles that best fit the stacked lensing cluster of Umetsu et al. (2011, strong-lensing selected). The blue dashed line shows an NFW profile that fits the stacked lensing cluster of Okabe et al. (2010b, X-ray selected). Horizontal lines indicate the critical density $\rho_{c}$ and $\bar{\rho}=0.3 \rho_{c}$. Bottom panels: cumulative density profiles of the ensemble clusters. Horizontal lines indicate enclosed densities of $(500,200,5.6) \rho_{c}$, where $\rho_{c}$ is evaluated at $z=0.16$, the median redshift of the HeCS sample.

(A color version of this figure is available in the online journal.)

Table 5

NFW Fits to HeCS Ensemble Mass Profiles

\begin{tabular}{lcccc}
\hline \hline Quartile & Median $^{\mathrm{a}}$ & Range $^{\mathrm{a}}$ & $\begin{array}{c}r_{200} \\
\left(h^{-1} \mathrm{Mpc}\right)\end{array}$ & $c_{200}$ \\
\hline$L_{X}: 1$ & 5.06 & $4.14-12.68$ & 1.48 & 7.3 \\
$L_{X}: 2$ & 2.96 & $2.33-3.71$ & 1.17 & 4.0 \\
$L_{X}: 3$ & 1.82 & $1.43-2.10$ & 1.07 & 2.5 \\
$L_{X}: 4$ & 1.11 & $0.62-1.27$ & 0.91 & 1.9 \\
\hline$M_{200}: 1$ & 7.84 & $4.95-12.40$ & 1.63 & 6.0 \\
$M_{200}: 2$ & 3.84 & $3.33-4.58$ & 1.44 & 8.8 \\
$M_{200}: 3$ & 2.44 & $1.92-3.10$ & 1.02 & 3.0 \\
$M_{200}: 4$ & 1.47 & $0.66-1.86$ & 0.95 & 3.2 \\
Umestu & 9.6 & $8.6-17.2$ & $\ldots$ & 6.28 \\
Okabe & 5.9 & $4.3-10.1$ & $\ldots$ & 2.93 \\
\hline
\end{tabular}

Note. ${ }^{a} L_{X} / E(z)$ is given in units of $10^{44} h^{-2} \mathrm{erg} \mathrm{s}^{-1}$ in the ROSAT band; $M_{200}$ is in units of $10^{14} h^{-1} M_{\odot}$.

NFW profile is consistent with the inner region of the ensemble cluster profile. Concentrations of $c \approx 3-5$ are predicted by numerical simulations of cluster-sized dark matter halos (e.g., Bullock et al. 2001). Further, Serra et al. (2011) show that cluster mass profiles in cosmological simulations follow the extrapolation of an NFW fit (performed on the inner $1 h^{-1} \mathrm{Mpc}$ using the caustic technique) out to $3-4 r_{200}$. That is, the simulated clusters follow NFW profiles well into their infall regions (see also Tavio et al. 2008).
Some investigators have suggested that the high concentration parameters found in cluster lensing profiles indicate problems with $\Lambda \mathrm{CDM}$ cosmology (e.g., Broadhurst et al. 2008). To ameliorate projection effects, Umetsu et al. (2011) constructed a stacked cluster and determined the mass profile from both strong and weak gravitational lensing. The magenta dashed line in Figure 11 shows an NFW profile with concentration $c=r_{200} / r_{s}=6.28$; this profile is an excellent fit to the stacked lensing cluster of Umetsu et al. (2011; K. Umetsu 2012, private communication), one of the highest-precision lensing profile estimates. Figure 11 also shows the best-fit NFW profile $(c=$ 2.93) from the stacked lensing cluster of Okabe et al. (2010b, K. Umetsu 2012, private communication) created from X-rayselected clusters. The median mass of the lensing-selected sample is slightly larger than the median mass of the highest- $M_{200}$ quartile, but the ranges overlap. The high-mass ensemble cluster of Okabe et al. (2010a) has similar $L_{X}$ and $M_{200}$ to our top quartile samples. The concentrations of the density profiles of the HeCS ensemble clusters generally lie between the lensing profile from the X-ray selected clusters and the profile from strong-lensing selected clusters (Table 5).

Our results for the ensemble $\mathrm{HeCS}$ clusters indicate qualitative agreement between the simulations and mass profiles determined from the caustic technique. Systematic uncertainties could be introduced by our assumption of constant $\mathcal{F}_{\beta}$, an assumption that overestimates the central masses of simulated clusters (Serra et al. 2011). Another possible concern in 


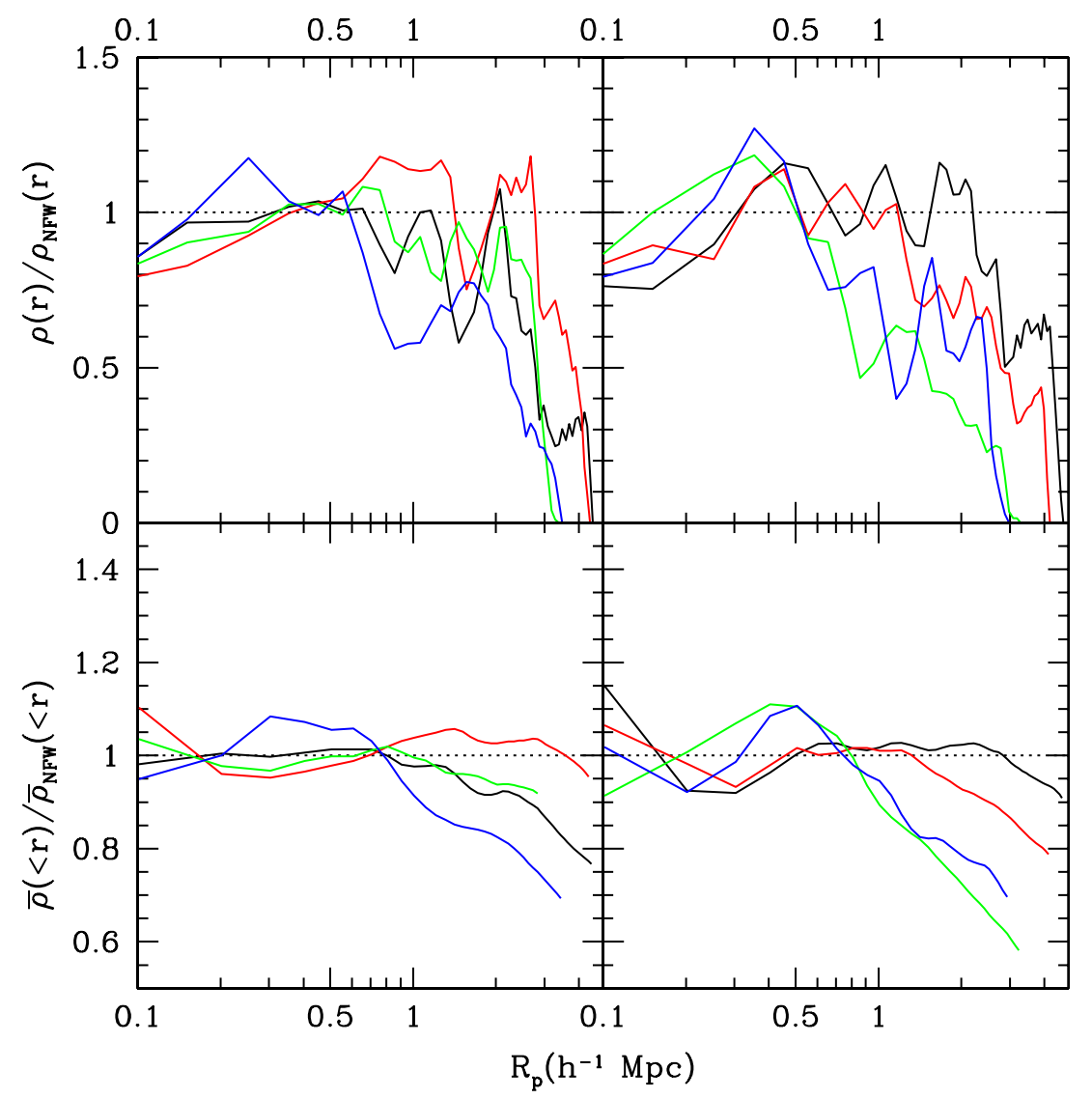

Figure 12. Residuals of NFW fits to the density profiles of ensemble clusters from Figure 11. Black, red, green, and blue solid lines show decreasing quartiles of $L_{X}$ (left panels) or $M_{200}$ (right panels). Top panels: residuals of the ensemble density profiles from NFW fits. Note that the NFW fits are performed with the mass profiles rather than the density profiles. Bottom panels: residuals of cumulative density profiles of the ensemble clusters from NFW fits to the inner $1 h^{-1} \mathrm{Mpc}$ (see Serra et al. 2011).

(A color version of this figure is available in the online journal.)

measuring $c$ for an ensemble cluster is that mis-centering could artificially smooth out the central density peak and thus artificially lower the measured concentration. The offset between the hierarchical centers we use and the X-ray centers is almost always less than $300 h^{-1} \mathrm{kpc}$, but Figure 11 shows that the two NFW models are very similar in the range $300-3000 h^{-1} \mathrm{kpc}$, suggesting that mis-centering could significantly impact concentrations from ensemble (or individual) caustic mass profiles. We will investigate possible systematic uncertainties in measuring concentrations with caustics in future work.

The lower panels of Figure 11 show the cumulative density profiles of the ensemble clusters. These profiles determine the values of the radii $r_{\Delta}$. For all quartiles, the caustic mass profiles extend well beyond $r_{200}$, but not quite to $r_{5.6}$ (the maximum radius of particles bound to the cluster in the far future). This limitation is primarily due to the limited radial extent of the HeCS data: a radius of $30^{\prime}$ corresponds to $4.2 h^{-1} \mathrm{Mpc}$ at $z=0.2$; the minimum value of $r_{5.6}$ for the highest $-L_{X}$ ensemble cluster is $5.8 h^{-1} \mathrm{Mpc}$.

Figure 12 shows the residuals from NFW fits to the inner $1 h^{-1} \mathrm{Mpc}$ of the ensemble clusters (fits performed on the caustic mass profile). Serra et al. (2011) show that this procedure yields accurate predictions of their simulated cluster mass profiles. The density profiles are noisy but seem to indicate that observed densities at large radii are slightly smaller than the densities of the extrapolated NFW fits. Table 5 lists the best-fit parameters. The NFW fits show a positive correlation between concentration and mass ( or $L_{X}$ ) contrary to the expected negative correlation (e.g., Bullock et al. 2001). Because the concentrations derived from caustic mass profiles may contain mass-dependent systematic uncertainties, we caution the reader that the trend evident in Table 5 could be dominated by systematic effects.

The lower panels of Figure 12 show that the cumulative density profiles agree within $\sim 20 \%$ of the extrapolated NFW profiles far beyond $r_{200}$. The smallest $-L_{X}$ quartile and the two smallest- $M_{200}$ quartiles have smaller cumulative densities than the extrapolated NFW profiles. We speculate that this deficit is a combination of the low- $c$ values of these profile fits $(c \sim 2-3)$ and a dearth of observed galaxies at large radii (Figure 10) resulting from the lower average redshifts of the constituent clusters and the finite field-of-view of Hectospec. Overall, the agreement between caustic mass profiles and NFW profiles extrapolated to large radius is excellent. This dynamical agreement is independent support for similar results derived from weak lensing profiles extending to large radius (Okabe et al. 2010a; Umetsu et al. 2011).

We use a pseudo-jackknife technique to quantify the statistical uncertainties in the ensemble density profiles. Specifically, we reanalyze the ensemble cluster for the highest $-L_{X}$ quartile in 13 subsamples where we remove one cluster from the ensemble for each subsample. Figure 13 shows the results of this test; the density profile has statistical uncertainties of $\lesssim 10 \%$ inside $r_{200}$ and $\sim 50 \%$ in the range $(1-4) r_{200}$. The enclosed density profile 


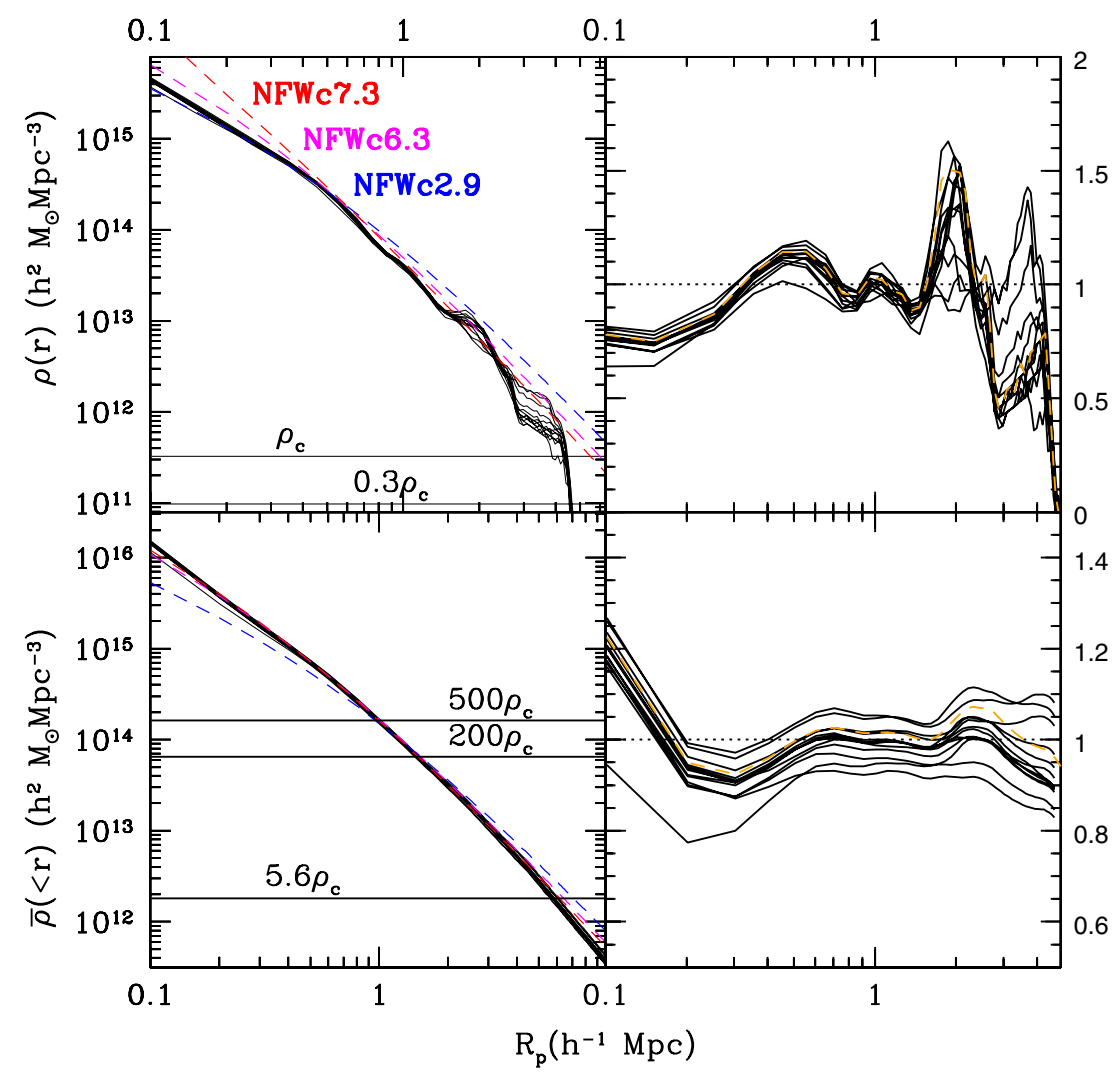

Figure 13. Density (top left) and enclosed density (bottom left) profiles of the highest- $L_{X}$ ensemble cluster determined from 13 pseudo-jackknife subsamples. Magenta, blue, and red dashed lines show NFW profiles with concentrations $c=6.3,2.9$, and 7.3, respectively (the red line is the best-fit to the enclosed density profile within $1 \mathrm{Mpc}$ ). Each subsample removes a different cluster from the sample. Right panels show the residuals from the best-fit NFW profile (fit within $1 h^{-1} \mathrm{Mpc}$ ). The orange dashed lines show the profiles determined from all clusters in the high- $L_{X}$ sample.

(A color version of this figure is available in the online journal.)

(or equivalently the mass profile) from the caustic technique has small $(\lesssim 10 \%)$ statistical uncertainties at $r<4 r_{200}$ (bottom right panel of Figure 13).

\section{PROPERTIES OF CLUSTER GALAXIES}

One goal of HeCS is to study the spectroscopic properties of galaxies in clusters and their infall regions. We defer a complete analysis to future work. Here, we investigate the possible impact of our target selection algorithm (which favors red-sequence galaxies) on the estimates of cluster mass profiles.

\subsection{Red Sequence Galaxies}

Because we targeted galaxies on the red sequence (Appendix), many of the cluster members lie on the red sequence. Figure 14 shows color-magnitude diagrams of cluster members. We computed $K$-corrections using the purely empirical $K$-corrections of Westra et al. (2010; these empirically based bandpass corrections naturally include evolutionary corrections). The red sequence of HeCS members can be described approximately as

$$
{ }^{0.0}(g-r)=-0.025\left(M_{r}+24\right)+0.87
$$

(solid line in Figure 14). One striking feature of Figure 14 is the small scatter in the red sequence. The outer lines are offset from the red sequence by $\pm 0.3 \mathrm{mag}$, approximately the color range where we assigned highest targeting priority. The inner lines are offset by $\pm 0.1 \mathrm{mag}$, demonstrating that most cluster members lie within this much narrower range of color. This result suggests

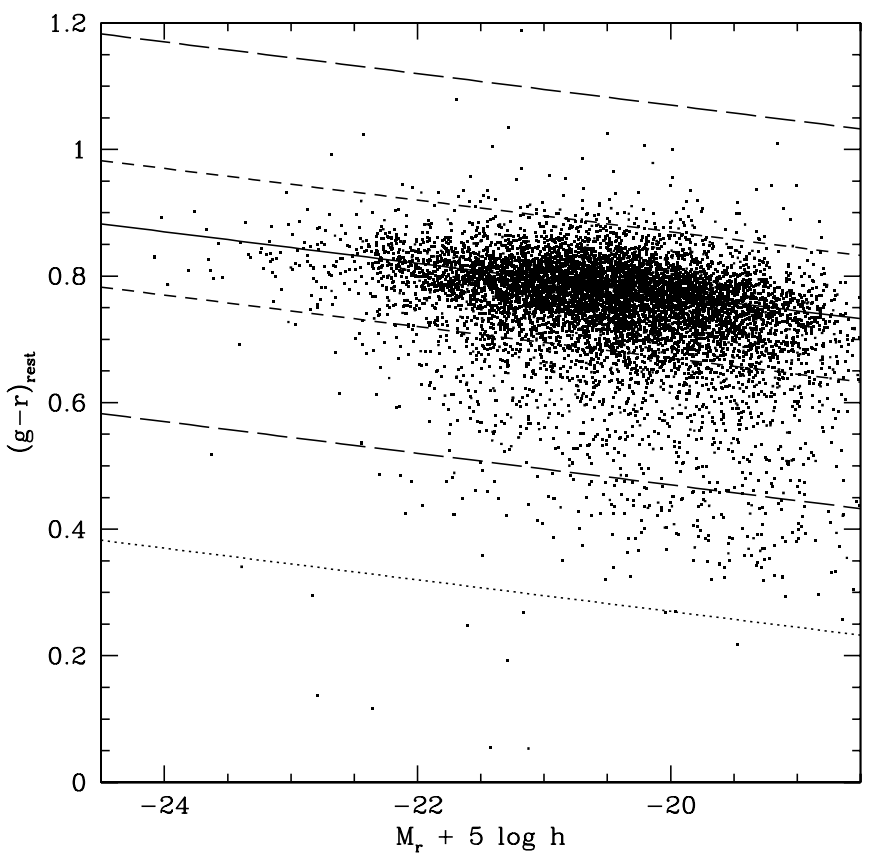

Figure 14. Color-magnitude diagram of member galaxies of the HeCS clusters including $K$-corrections. Long-dashed lines show $0.3 \mathrm{mag}$ away from the red sequence, approximately the limits of our priority target selection. Most of the HeCS members are within $0.1 \mathrm{mag}$ of the red sequence (short-dashed lines), indicating that the color selection includes the vast majority of red-sequence cluster galaxies. The dotted line shows the color range $0.2 \mathrm{mag}$ blueward of the red-sequence cut used to select additional targets to fill unused fibers. 


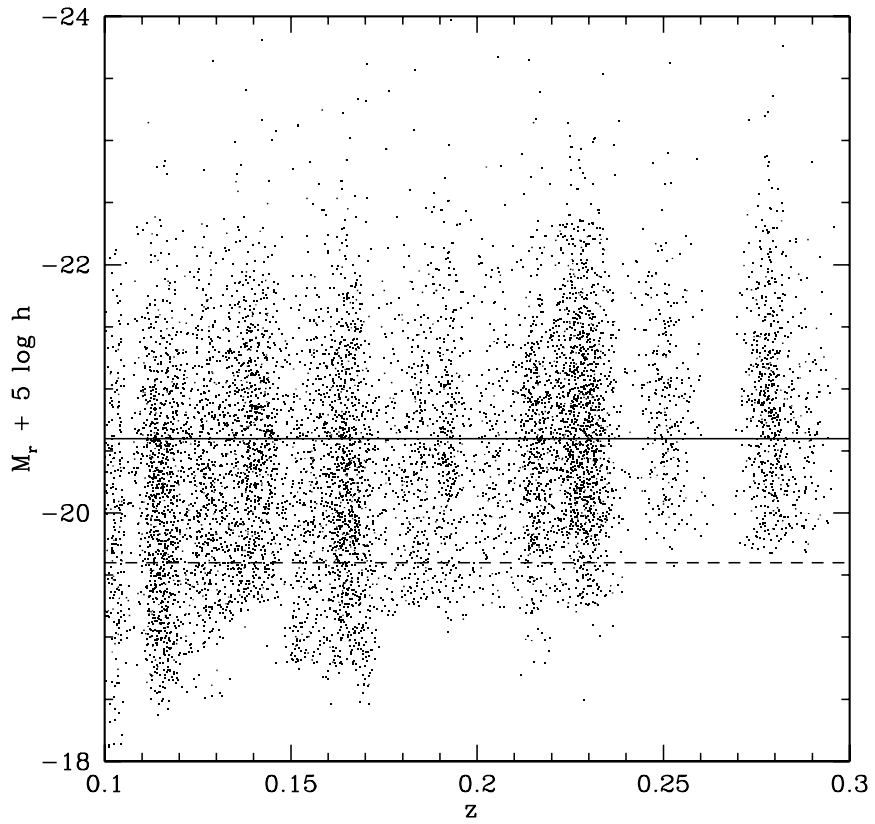

Figure 15. Absolute magnitude vs. clustrocentric radius for cluster members in the $\mathrm{HeCS}$ clusters. Solid and dashed lines indicate absolute magnitudes of $M_{r}^{*}$ and $M_{r}^{*}+1$, respectively. that the galaxy properties are very similar and that the SDSS photometric uncertainties are minimal. Lines with a slope of -0.04 (observed colors) or -0.025 (rest-frame colors) provide a remarkably accurate description of the red sequences.

Figure 15 shows the absolute magnitudes of cluster members versus their redshifts. The solid line shows $M_{r}=-20.60+$ $5 \log h$, approximately the characteristic magnitude $M_{r}^{*}$ of the luminosity function of field galaxies in SDSS (Blanton et al. 2003a). The dashed line is one magnitude fainter $\left(M_{r}^{*}+1\right)$, a luminosity limit often adopted for describing galaxies (e.g., Tinker et al. 2005). CIRS shows that this limit is the minimum depth for a cluster sample that yields enough members to identify caustics. Figure 15 shows that all HeCS clusters at $z<0.25$ meet this condition. The remaining clusters are still well-sampled because these clusters have larger X-ray luminosities (and hence more bright member galaxies) than the CIRS clusters (Figure 1).

\subsection{Extremely Red Cluster Galaxies}

Figure 16 shows that very few cluster members have $g-r$ colors significantly redder than the red sequence. In fact, all of the five cluster members in RXJ2129 and A2261 with colors redder than the red-sequence cut have incorrect colors due to their proximity to nearby bright stars or galaxies. Using fiber

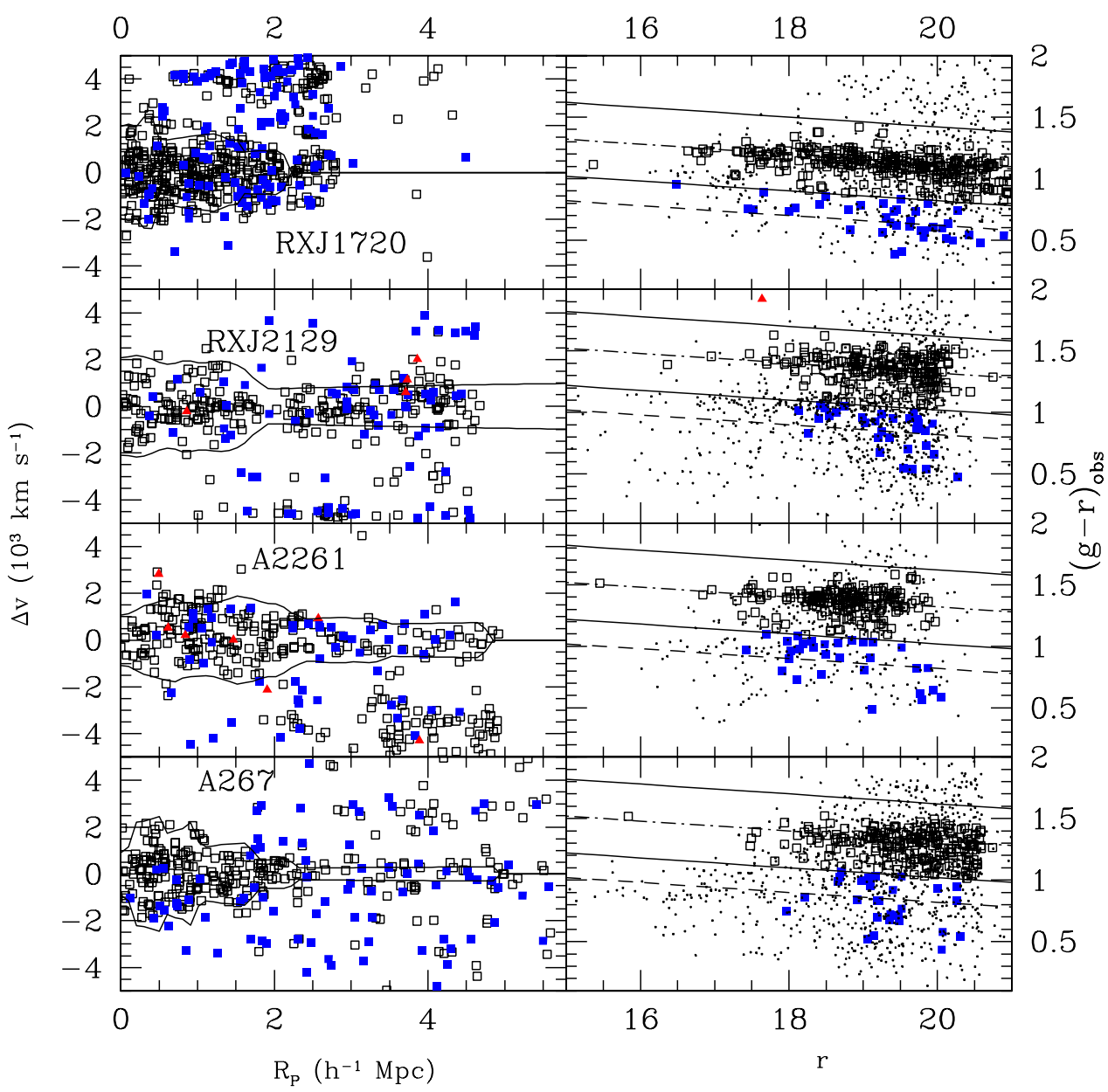

Figure 16. Left: redshift (rest-frame clustrocentric velocity) vs. radius for galaxies in RXJ1720, RXJ2129, A2261, and A267. For these clusters, spectroscopic redshifts are available for a wide range of colors (see Section 2.3). Open squares are red sequence galaxies (within $0.3 \mathrm{mag}$ ), solid blue squares are blue cloud galaxies, and red triangles are more than 0.3 mag redder than the red sequence. Right: color-magnitude diagrams of the four clusters. Large squares are cluster members (as defined by the caustics) and small dots are non-members.

(A color version of this figure is available in the online journal.) 
magnitudes, all six have $g-r$ colors below the red-sequence cutoff.

Because the red sequence represents some of the oldest known stellar populations, colors redder than the red sequence would most likely arise from extreme dust reddening. Thus, the absence of cluster members with colors more than 0.3 mag redder than the red sequence indicates that very few cluster members are extremely reddened.

\subsection{Blue Cluster Galaxies: Are They Common? Do They Affect Dynamical Mass Estimates?}

To test the sensitivity of our dynamical estimates on the red-sequence target selection, we observed several Hectospec pointings in the clusters A267, A2261, and RXJ2129 (all at $z \approx 0.23$ ) to sample blue galaxies. The Hectospec survey of RXJ1720 (Owers et al. 2011) also samples both red-sequence and blue galaxies. Figure 16 shows that very few blue galaxies are cluster members, consistent with previous studies (e.g., Dressler 1980; Rines et al. 2005). The solid lines show the approximate limits of the HeCS priority target selection used for the other HeCS clusters. The dashed line, slightly bluer, indicates our limit for low-priority targets. Very few galaxies below the solid lines (filled blue squares in the left panels) are cluster members. Combining the four clusters, 138 of 1053 cluster members $(13.1 \%)$ lie blueward of our red-sequence cut. A two-sample K-S test indicates that the velocity distributions of red and blue galaxies are consistent with being drawn from the same parent population. Further, the velocity dispersion of the ensemble cluster (all galaxies) is only $0.8 \%$ larger than the velocity dispersion of the red-sequence galaxies. Limiting the sample to galaxies inside $r_{200}, 38$ of 488 members $(7.8 \%)$ lie blueward of the red-sequence cut; including non-red-sequence galaxies increases the velocity dispersion by $0.3 \%$. We thus conclude that targeting red-sequence galaxies produces no significant bias in our estimates of dynamical masses or velocity dispersions. Previous claims of velocity segregation often relied on much smaller samples where membership classification may have been less robust. Serra \& Diaferio (2012) discusses the completeness of galaxy samples identified using the caustic technique in simulations. They conclude that the caustics identify most of the members and include few interlopers.

Mahajan et al. (2011) recently studied the dependence of cluster galaxy properties on their projected velocity relative to the cluster center. They bin galaxies both by projected radius and by velocity offset (e.g., galaxies with $|\Delta v|=$ $\left.(0-1) \sigma_{p}, 1-2 \sigma_{p}, 2-3 \sigma_{p}\right)$. At fixed projected radius, their Figure 3 shows little dependence of the color distribution on velocity offset, suggesting that the velocity distribution does not depend dramatically on galaxy color, consistent with our results.

Our Hectospec (and SDSS) redshifts show that some cluster members are "blue cloud" galaxies with colors indicating recent star formation (Figure 16). The fraction of "blue cloud" galaxies seems to increase with increasing absolute magnitude, similar to field galaxies (e.g., Blanton et al. 2003b). This conclusion could be tested with more extensive spectroscopic sampling of faint galaxies that lie blueward of the HeCS red sequence cuts.

\section{CONCLUSIONS}

We present 22,680 redshifts from the Hectospec Cluster Survey (HeCS). HeCS is a MMT/Hectospec spectroscopic survey of X-ray-selected clusters contained in the imaging footprint of SDSS DR6. Our redshifts confirm that infall patterns known as "caustics" are clearly present in X-ray clusters at moderate redshift. Combined with SDSS data, we define a sample of 10,145 cluster members.

We use the infall patterns to compute mass profiles for the clusters extending in many cases to the turnaround radius of the cluster. In numerical simulations of a $\Lambda \mathrm{CDM}$ universe, the mass within the radius $r_{5.6}$ (the average density inside $r_{5.6}$ is $5.6 \rho_{c}(z)$ ) is approximately equal to the ultimate mass of the cluster at late times. These simulations predict that this ultimate halo mass is $\approx 2.2 M_{200}$. The HeCS mass profiles provide an observational estimate of $M_{5.6}=(1.99 \pm 0.11) M_{200}$, in excellent agreement with the predictions.

The caustic technique enables a unique measure of the large-scale behavior of cluster mass profiles. The striking agreement with the theoretical predictions (Nagamine \& Loeb 2003; Busha et al. 2005; Dünner et al. 2006) is an interesting new test of models for the growth of structure in the $\Lambda \mathrm{CDM}$ cosmology.

The ensemble clusters we construct here average over projection effects. The density profiles of these ensemble clusters closely resemble NFW profiles out to radii of at least $2 r_{200}$. The NFW concentrations of our ensemble clusters are similar to or slightly larger than the predictions of simulations. Systematic uncertainties from projection effects and from determining the cluster prevent us from confirming or rejecting the "over-concentration" problem of strong-lensing clusters (e.g., Broadhurst \& Barkana 2008). Future work will test the precision and accuracy of measurements of NFW concentrations from caustic mass profiles and compare these measurements to predictions from numerical simulations of dark matter halos.

The CAIRNS and CIRS projects demonstrated that caustic patterns are present in nearly all rich, X-ray luminous galaxy clusters. Here we show that these patterns are also prominent in clusters at $z=0.1-0.3$. The ensemble clusters we construct enhance the visibility of these patterns.

The HeCS clusters show a relation between $L_{X}$ and $\sigma_{p}$ (we will investigate the specific relation in future work); we show that outliers in this relation can be attributed to contamination from X-ray point sources (A689) or strong cooling cores (RXJ1504). The scaling relation $M_{200}-\sigma_{p}$ between virial masses and line-of-sight velocity dispersions is in excellent agreement with the scaling relation of dark matter particles in simulated clusters (Evrard et al. 2008). Other projects planned with HeCS are investigations of cluster scaling relations between galaxy dynamics and other mass probes (richness, $Y_{X}, Y_{\mathrm{SZ}}, M_{\mathrm{lens}}$; see Rines et al. 2010), a determination of the mass function of clusters (e.g., Rines et al. 2008; Vikhlinin et al. 2009; Mantz et al. 2008; Henry et al. 2009), and a detailed study of the photometric and spectroscopic properties of cluster galaxies. A companion paper (Geller et al. 2013) compares caustic mass profiles to those determined from weak gravitational lensing, the only other mass estimator that applies to the non-virialized infall regions surrounding clusters.

K.R. was funded by a Cottrell College Science Award from the Research Corporation. A.D. acknowledges partial support from the INFN grant PD51 and the PRIN-MIUR-2008 grant "Matter-antimatter asymmetry, dark matter and dark energy in the LHC Era." Research support for M.J.G. and M.J.K. is provided by the Smithsonian Institution. We thank YuYing Zhang, Keiichi Umetsu, Rien van de Weygaert, and the anonymous referee for helpful comments and suggestions. 
Funding for the Sloan Digital Sky Survey (SDSS) has been provided by the Alfred P. Sloan Foundation, the Participating Institutions, the National Aeronautics and Space Administration, the National Science Foundation, the U.S. Department of Energy, the Japanese Monbukagakusho, and the Max Planck Society. The SDSS Web site is http://www.sdss.org/. The SDSS is managed by the Astrophysical Research Consortium (ARC) for the Participating Institutions. The Participating Institutions are The University of Chicago, Fermilab, the Institute for Advanced Study, the Japan Participation Group, The Johns Hopkins University, the Korean Scientist Group, Los Alamos National Laboratory, the Max-Planck-Institute for Astronomy (MPIA), the Max-Planck-Institute for Astrophysics (MPA), New Mexico State University, University of Pittsburgh, University of Portsmouth, Princeton University, the United States Naval Observatory, and the University of Washington.

Facility: MMT (Hectospec)

\section{APPENDIX}

\section{SPECTROSCOPIC TARGET SELECTION}

We selected targets for spectroscopy based on photometry from SDSS DR6. First, we extracted galaxy catalogs within $32^{\prime}$ from the SDSS SQL server. ${ }^{7}$ For each cluster, we identify the red sequence from a color-magnitude diagram using $g-r$ colors and $r$-band apparent magnitudes. Following guidance from the SDSS web pages, ${ }^{8}$ we adopt the composite model magnitudes for our apparent magnitudes and colors. Composite model magnitudes are a linear combination of the best-fit exponential and de Vaucouleurs model profiles. Composite model magnitudes should have higher signal-to-noise than Petrosian magnitudes, especially for relatively faint galaxies such as those studied here.

To identify the red sequence, we used a fixed slope of -0.04 mag $\mathrm{mag}^{-1}$ for all clusters and chose the intercept based on visual inspection of the color-magnitude diagram. ${ }^{9}$ To eliminate some stars and artifacts (e.g., portions of diffraction spikes from bright stars), we restrict the sample to galaxies with $r$-band surface brightness brighter than 22.9 mag $\operatorname{arcsec}^{-2}$ (we later inspect objects with fainter surface brightnesses to include some galaxies eliminated by this cut).

The Hectospec fiber assignment software $x$ fitfibs ${ }^{10}$ allows the user to assign rankings to targets. If we ignored the spatial positions of our targets, fiber collisions would prevent many objects in the central portion of the field (usually the center of the cluster) from being observed. We therefore assigned highest priority to galaxies brighter than a cluster-dependent limiting magnitude within \pm 0.3 magnitudes of the red sequence and within 2:4 of the X-ray center. We give second priority to red galaxies near the center and up to one magnitude fainter. Priorities are then assigned in annuli (outer radii 7.5 and $15^{\prime}$ ) according to apparent magnitude. In general, our target catalogs contained approximately twice as many targets as the number of fibers available. Most bright targets in the inner $15^{\prime}$ are assigned fibers, as are many fainter targets in this region. Unassigned targets are typically in the outer parts of the Hectospec field,

\footnotetext{
http://cas.sdss.org/astrodr6/en/tools/search/sql.asp

8 http://www.sdss.org/dr6/algorithms/photometry.html

9 For eight clusters observed in 2007 (A2111, A2187, A2219, A2259, A2396, A2631, A2645, and RXJ2129), we used a simple $g-r$ color cut rather than a red-sequence cut. This small sampling difference has no significant impact on our results.

10 https://www.cfa.harvard.edu/ john/xfitfibs/
}

with a modest bias against targets in local overdensities due to fiber collisions. Later analysis shows that the $\pm 0.3 \mathrm{mag}$ band around the red sequence is significantly larger than the actual thickness of the red sequence (see Section 5.1); thus, the precise choice of red-sequence intercept has essentially no impact on our results. Because cluster members are centrally concentrated, we found that prioritizing central objects is critical to obtain reasonably uniform sampling as a function of radius.

Because fiber placement constraints would sometimes prevent fibers from being deployed, we add galaxies up to 0.2 magnitude bluer than our red-sequence cutoff to the target list. We use the SDSS Image List tool ${ }^{11}$ to visually inspect all targets prior to observation. This visual inspection identifies artifacts and bright stars. We added a small number of targets that are not identified as separate objects by the DR6 photometric pipeline (these targets are usually merged with a nearby star or galaxy).

We observed two Hectospec pointings per cluster for clusters at $z>0.15$ and one pointing per cluster for clusters at $z=$ $0.10-0.15$. We adopted this strategy because SDSS redshifts of Main Sample galaxies at $r<17.77$ provide many members for the lower-redshift clusters (although not enough to enable a full caustic analysis). Also, the lower-redshift clusters have generally smaller X-ray luminosities (because HeCS is flux-limited) and thus have smaller expected masses. Combined with the larger angular size of these clusters, one Hectospec pointing combined with SDSS redshifts is generally sufficient to avoid sampling bias. For clusters at $z>0.15$, we chose apparent magnitude limits for each cluster to select 900-1300 candidate targets for two Hectospec pointings (potentially up to 600 targets). The bright magnitude limit for these clusters is $18.5<r<20.0$, corresponding to $M_{r}^{*}+1$ or fainter for most clusters (Figure 15). For clusters at $z=0.10-0.15$, we chose apparent magnitude limits for each cluster to select 500-600 candidate targets for a single Hectospec pointing (potentially up to 300 targets). The bright magnitude limit for these clusters is $17.8<r<18.8$, again corresponding to $M_{r}^{*}+1$ or fainter for most clusters (Figure 15).

\section{REFERENCES}

Adelman-McCarthy, J. K., Agüeros, M. A., Allam, S. S., et al. 2008, ApJS, 175,297

Barrena, R., Boschin, W., Girardi, M., \& Spolaor, M. 2007, A\&A, 467, 37 Biviano, A., \& Girardi, M. 2003, ApJ, 585, 205

Blanton, M. R., Hogg, D. W., Bahcall, N. A., et al. 2003a, ApJ, 592, 819 Blanton, M. R., Hogg, D. W., Bahcall, N. A., et al. 2003b, ApJ, 594, 186 Böhringer, H., Burwitz, V., Zhang, Y.-Y., Schuecker, P., \& Nowak, N. 2005, ApJ, 633, 148

Böhringer, H., Collins, C. A., Guzzo, L., et al. 2002, ApJ, 566, 93

Böhringer, H., Schuecker, P., Guzzo, L., et al. 2004, A\&A, 425, 367

Böhringer, H., Voges, W., Huchra, J. P., et al. 2000, ApJS, 129, 435

Boschin, W., Girardi, M., Barrena, R., \& Nonino, M. 2012, A\&A, 540, A43

Boschin, W., Girardi, M., Barrena, R., et al. 2004, A\&A, 416, 839

Broadhurst, T., Takada, M., Umetsu, K., et al. 2005, ApJL, 619, L143

Broadhurst, T., Umetsu, K., Medezinski, E., Oguri, M., \& Rephaeli, Y. 2008, ApJL, 685, L9

Broadhurst, T. J., \& Barkana, R. 2008, MNRAS, 390, 1647

Bullock, J. S., Kolatt, T. S., Sigad, Y., et al. 2001, MNRAS, 321, 559

Busha, M. T., Adams, F. C., Wechsler, R. H., \& Evrard, A. E. 2003, ApJ, 596, 713

Busha, M. T., Evrard, A. E., Adams, F. C., \& Wechsler, R. H. 2005, MNRAS, 363, L11

Carlberg, R. G., Yee, H. K. C., \& Ellingson, E. 1997, ApJ, 478, 462

Carlberg, R. G., Yee, H. K. C., Ellingson, E., et al. 1996, ApJ, 462, 32

Coe, D., Umetsu, K., Zitrin, A., et al. 2012, ApJ, 757, 22

$\overline{11 \text { http://cas.sdss.org/dr6/en/tools/chart/list.asp }}$ 
Cross, N. J. G., Driver, S. P., Liske, J., et al. 2004, MNRAS, 349, 576

Czoske, O. 2004, in IAU Colloq. 195, Outskirts of Galaxy Clusters: Intense Life in the Suburbs, ed. A. Diaferio (Cambridge: Cambridge Univ. Press), 183

Danese, L., de Zotti, G., \& di Tullio, G. 1980, A\&A, 82, 322

David, L. P., \& Kempner, J. 2004, ApJ, 613, 831

Diaferio, A. 1999, MNRAS, 309, 610

Diaferio, A., \& Geller, M. J. 1997, ApJ, 481, 633

Diaferio, A., Geller, M. J., \& Rines, K. J. 2005, ApJL, 628, L97

Diemand, J., Moore, B., \& Stadel, J. 2004, MNRAS, 352, 535

Dressler, A. 1980, ApJ, 236, 351

Drinkwater, M. J., Gregg, M. D., \& Colless, M. 2001, ApJ, 548, L139

Dünner, R., Araya, P. A., Meza, A., \& Reisenegger, A. 2006, MNRAS, 366, 803

Ebeling, H., Edge, A. C., Allen, S. W., et al. 2000, MNRAS, 318, 333

Ebeling, H., Edge, A. C., Bohringer, H., et al. 1998, MNRAS, 301, 881

Eke, V. R., Cole, S., \& Frenk, C. S. 1996, MNRAS, 282, 263

Evrard, A. E., Bialek, J., Busha, M., et al. 2008, ApJ, 672, 122

Fabricant, D., Fata, R., Roll, J., et al. 2005, PASP, 117, 1411

Faltenbacher, A., Kravtsov, A. V., Nagai, D., \& Gottlöber, S. 2005, MNRAS, 358,139

Geller, M. J., Diaferio, A., \& Kurtz, M. J. 1999, ApJL, 517, L23

Geller, M. J., Diaferio, A., \& Kurtz, M. J. 2011, AJ, 142, 133

Geller, M. J., Diaferio, A., Kurtz, M. J., Dell'Antonio, I. P., \& Fabricant, D. G. 2012, AJ, 143, 102

Geller, M. J., Diaferio, A., Rines, K. J., \& Serra, A. L. 2013, ApJ, 764, 58

Giles, P. A., Maughan, B. J., Birkinshaw, M., Worrall, D. M., \& Lancaster, K. 2012, MNRAS, 419, 503

Giovannini, G., Tordi, M., \& Feretti, L. 1999, NewA, 4, 141

Girardi, M., Giuricin, G., Mardirossian, F., Mezzetti, M., \& Boschin, W. 1998, ApJ, 505, 74

Gladders, M. D., \& Yee, H. K. C. 2000, AJ, 120, 2148

Govoni, F., Markevitch, M., Vikhlinin, A., et al. 2004, ApJ, 605, 695

Gramann, M., \& Suhhonenko, I. 2002, MNRAS, 337, 1417

Haines, C. P., Smith, G. P., Egami, E., et al. 2009, MNRAS, 396, 1297

Haines, C. P., Smith, G. P., Pereira, M. J., et al. 2010, A\&A, 518, L19

Hallman, E. J., Motl, P. M., Burns, J. O., \& Norman, M. L. 2006, ApJ, 648, 852

Henry, J. P., Evrard, A. E., Hoekstra, H., Babul, A., \& Mahdavi, A. 2009, ApJ, 691, 1307

Henry, J. P., Gioia, I. M., Maccacaro, T., et al. 1992, ApJ, 386, 408

Hoekstra, H., Hartlap, J., Hilbert, S., \& van Uitert, E. 2011, MNRAS, 412, 2095

Kaiser, N. 1987, MNRAS, 227, 1

Kauffmann, G., Colberg, J. M., Diaferio, A., \& White, S. D. M. 1999a, MNRAS, 303, 188

Kauffmann, G., Colberg, J. M., Diaferio, A., \& White, S. D. M. 1999b, MNRAS, 307,529

Koranyi, D. M., \& Geller, M. J. 2000, AJ, 119, 44

Kravtsov, A. V., Vikhlinin, A., \& Nagai, D. 2006, ApJ, 650, 128

Kurtz, M. J., \& Mink, D. J. 1998, PASP, 110, 934

Lemze, D., Broadhurst, T., Rephaeli, Y., Barkana, R., \& Umetsu, K. 2009, ApJ, 701,1336

Lin, Y., Mohr, J. J., \& Stanford, S. A. 2004, ApJ, 610, 745

Łokas, E. L., \& Mamon, G. A. 2003, MNRAS, 343, 401

Łokas, E. L., Prada, F., Wojtak, R., Moles, M., \& Gottlöber, S. 2006, MNRAS, 366, L26

Mahajan, S., Mamon, G. A., \& Raychaudhury, S. 2011, MNRAS, 416, 2882

Mahdavi, A., Trentham, N., \& Tully, R. B. 2005, AJ, 130, 1502
Majumdar, S., \& Mohr, J. J. 2004, ApJ, 613, 41

Mamon, G. A., Biviano, A., \& Boué, G. 2013, MNRAS, 429, 3079

Mantz, A., Allen, S. W., Ebeling, H., \& Rapetti, D. 2008, MNRAS, 387, 1179

Maughan, B. J., Jones, C., Forman, W., \& Van Speybroeck, L. 2008, ApJS, 174,117

Nagai, D., Vikhlinin, A., \& Kravtsov, A. V. 2007, ApJ, 655, 98

Nagamine, K., \& Loeb, A. 2003, NewA, 8, 439

Navarro, J. F., Frenk, C. S., \& White, S. D. M. 1997, ApJ, 490, 493

Okabe, N., Takada, M., Umetsu, K., Futamase, T., \& Smith, G. P. 2010a, PASJ, 62,811

Okabe, N., \& Umetsu, K. 2008, PASJ, 60, 345

Okabe, N., Zhang, Y.-Y., Finoguenov, A., et al. 2010b, ApJ, 721, 875

Owers, M. S., Nulsen, P. E. J., \& Couch, W. J. 2011, ApJ, 741, 122

Pimbblet, K. A., Smail, I., Edge, A. C., et al. 2006, MNRAS, 366, 645

Postman, M., Coe, D., Benítez, N., et al. 2012, ApJS, 199, 25

Ragozzine, B., Clowe, D., Markevitch, M., Gonzalez, A. H., \& Bradač, M. 2012, ApJ, 744, 94

Regös, E., \& Geller, M. J. 1989, AJ, 98, 755

Reiprich, T. H., \& Böhringer, H. 2002, ApJ, 567, 716

Reisenegger, A., Quintana, H., Carrasco, E. R., \& Maze, J. 2000, AJ, 120, 523

Rines, K., \& Diaferio, A. 2006, AJ, 132, 1275

Rines, K., \& Diaferio, A. 2010, AJ, 139, 580

Rines, K., Diaferio, A., \& Natarajan, P. 2008, ApJL, 679, L1

Rines, K., Geller, M. J., \& Diaferio, A. 2010, ApJL, 715, L180

Rines, K., Geller, M. J., Diaferio, A., Kurtz, M. J., \& Jarrett, T. H. 2004, AJ, 128,1078

Rines, K., Geller, M. J., Diaferio, A., et al. 2002, AJ, 124, 1266

Rines, K., Geller, M. J., Diaferio, A., Mohr, J. J., \& Wegner, G. A. 2000, AJ, 120, 2338

Rines, K., Geller, M. J., Kurtz, M. J., \& Diaferio, A. 2003, AJ, 126, 2152

Rines, K., Geller, M. J., Kurtz, M. J., \& Diaferio, A. 2005, AJ, 130, 1482

Rines, K., Mahdavi, A., Geller, M. J., et al. 2001, ApJ, 555, 558

Santos, W. A., Mendes de Oliveira, C., \& Sodré, L., Jr. 2007, AJ, 134, 1551

Serra, A. L., \& Diaferio, A. 2012, arXiv:1211.3669

Serra, A. L., Diaferio, A., Murante, G., \& Borgani, S. 2011, MNRAS, 412, 800

Smail, I., Hogg, D. W., Blandford, R., et al. 1995, MNRAS, 277, 1

Stoughton, C., Lupton, R. H., Bernardi, M., et al. 2002, AJ, 123, 485

Strauss, M. A., Weinberg, D. H., Lupton, R. H., et al. 2002, AJ, 124, 1810

Tavio, H., Cuesta, A. J., Prada, F., Klypin, A. A., \& Sanchez-Conde, M. A. 2008, arXiv:0807.3027

The, L. S., \& White, S. D. M. 1986, AJ, 92, 1248

Tinker, J. L., Weinberg, D. H., Zheng, Z., \& Zehavi, I. 2005, ApJ, 631, 41

Umetsu, K., Broadhurst, T., Zitrin, A., Medezinski, E., \& Hsu, L.-Y. 2011, ApJ, 729,127

van der Marel, R. P., Magorrian, J., Carlberg, R. G., Yee, H. K. C., \& Ellingson, E. 2000, AJ, 119, 2038

van Haarlem, M., \& van de Weygaert, R. 1993, ApJ, 418, 544

Vikhlinin, A., Burenin, R. A., Ebeling, H., et al. 2009, ApJ, 692, 1033

Voges, W., Aschenbach, B., Boller, Th., et al. 1999, A\&A, 349, 389

Westra, E., Geller, M. J., Kurtz, M. J., Fabricant, D. G., \& Dell'Antonio, I. 2010, PASP, 122, 1258

Zhang, Y.-Y., Andernach, H., Caretta, C. A., et al. 2011, A\&A, 526, A105

Zhang, Y.-Y., Verdugo, M., Klein, M., \& Schneider, P. 2012, A\&A, 542, A106

Zwicky, F. 1933, AcHPh, 6, 110

Zwicky, F. 1937, ApJ, 86, 217 